\title{
Exponential Separations in the Energy Complexity of Leader Election
}

\author{
YI-JUN CHANG, University of Michigan, USA \\ TSVI KOPELOWITZ, Bar-Ilan University, Israel \\ SETH PETTIE, University of Michigan, USA \\ RUOSONG WANG, Carnegie Mellon University, USA \\ WEI ZHAN, Princeton University, USA
}

Energy is often the most constrained resource for battery-powered wireless devices, and most of the energy is often spent on transceiver usage (i.e., transmitting and receiving packets) rather than computation. In this article, we study the energy complexity of fundamental problems in several models of wireless radio networks. It turns out that energy complexity is very sensitive to whether the devices can generate random bits and their ability to detect collisions. We consider four collision detection models: Strong-CD (in which transmitters and listeners detect collisions), Sender-CD (in which only transmitters detect collisions), Receiver-CD (in which only listeners detect collisions), and No-CD (in which no one detects collisions).

The take-away message of our results is quite surprising. For randomized algorithms, there is an exponential gap between the energy complexity of Sender-CD and Receiver-CD:

$$
\text { Randomized: No-CD = Sender-CD } \gg \text { Receiver-CD = Strong-CD }
$$

and for deterministic algorithms, there is another exponential gap in energy complexity, but in the reverse direction:

$$
\text { Deterministic: No-CD = Receiver-CD } \gg \text { Sender-CD = Strong-CD }
$$

Precisely, the randomized energy complexity of Leader Election is $\Theta\left(\log ^{*} n\right)$ in Sender-CD but $\Theta\left(\log \left(\log ^{*} n\right)\right)$ in Receiver-CD, where $n$ is the number of devices, which is unknown to the devices at the beginning; the deterministic complexity of Leader Election is $\Theta(\log N)$ in Receiver-CD but $\Theta(\log \log N)$ in Sender-CD, where $N$ is the size of the ID space.

There is a tradeoff between time and energy. We provide a new upper bound on the time-energy tradeoff curve for randomized algorithms. A critical component of this algorithm is a new deterministic Leader Election algorithm for dense instances, when $n=\Theta(N)$, with inverse Ackermann energy complexity.

\section{CCS Concepts: • Theory of computation $\rightarrow$ Distributed algorithms;}

Additional Key Words and Phrases: Energy complexity, leader election, wireless network

A preliminary version of this article [11] was presented at the 49th Annual ACM Symposium on the Theory of Computing (STOC) June 19-23, 2017. Supported by NSF grants CNS-1318294, CCF-1514383, CCF-1637546, and CCF-1815316. Research performed while Ruosong Wang and Wei Zhan were visiting University of Michigan. Ruosong Wang and Wei Zhan are supported in part by the National Basic Research Program of China, grants 2015CB358700, 2011CBA00300, 2011CBA00301 and the National Natural Science Foundation of China, grants 61202009, 61033001, 61361136003.

Authors' addresses: Y.-J. Chang and S. Pettie, University of Michigan, Ann Arbor, MI; emails: \{cyijun, pettie\}@umich.edu; T. Kopelowitz, Bar-Ilan University, Ramat Gan, Israel; email: kopelot@gmail.com; R. Wang, Carnegie Mellon University, Pittsburgh, PA; email: ruosongw@andrew.cmu.edu; W. Zhan, Princeton University, Princeton, NJ; email: weizhan@cs. princeton.edu.

Permission to make digital or hard copies of all or part of this work for personal or classroom use is granted without fee provided that copies are not made or distributed for profit or commercial advantage and that copies bear this notice and the full citation on the first page. Copyrights for components of this work owned by others than the author(s) must be honored. Abstracting with credit is permitted. To copy otherwise, or republish, to post on servers or to redistribute to lists, requires prior specific permission and/or a fee. Request permissions from permissions@acm.org.

(C) 2019 Copyright held by the owner/author(s). Publication rights licensed to ACM.

1549-6325/2019/10-ART49 \$15.00

https://doi.org/10.1145/3341111 


\section{ACM Reference format:}

Yi-Jun Chang, Tsvi Kopelowitz, Seth Pettie, Ruosong Wang, and Wei Zhan. 2019. Exponential Separations in the Energy Complexity of Leader Election. ACM Trans. Algorithms 15, 4, Article 49 (October 2019), 31 pages. https://doi.org/10.1145/3341111

\section{INTRODUCTION}

In many networks of wireless devices the scarcest resource is energy, and the lion's share of energy is often spent on radio transceiver usage [4,39,43,45]-transmitting and receiving packets-not on computation per se. In this article, we investigate the energy complexity of fundamental problems in synchronized single-hop wireless networks: Leader Election, Approximate Counting, and taking a Census.

In all models, we consider time to be partitioned into discrete slots; all devices have access to a single shared channel and can choose, in each time slot, to either transmit a message $m$ from some space $\mathcal{M}$, listen to the channel, or remain idle. Transmitting and listening each cost one unit of energy; we measure the energy usage of an algorithm on $n$ devices by the worst case energy usage of any device. For the sake of simplicity, we assume computation is free and the message size is unbounded. If exactly one device transmits, all listeners hear the message $m$, and if zero devices transmit, all listeners hear a special message $\lambda_{S}$ indicating silence. We consider four collision detection models depending on whether transmitters and listeners can detect collisions.

Strong-CD. Each transmitter and listener receives one of three signals: $\lambda_{S}$ (silence, if zero devices transmit), $\lambda_{N}$ (noise, if $\geq 2$ devices transmit), or a message $m \in \mathcal{M}$ (if one device transmits).

Sender-CD. (Often called "No-CD” [27]) Each transmitter and listener receives one of two signals: $\lambda_{S}$ (zero or $\geq 2$ devices transmit), or a message $m \in \mathcal{M}$ (if one device transmits). Observe that the Sender-CD model has no explicit collision detection but still allows for sneaky collision detection: if a sender hears $\lambda_{S}$, it can infer that there was at least one other sender.

Receiver-CD. Transmitters receive no signal. Each listener receives one of three signals: $\lambda_{S}$ (silence, if zero devices transmit), $\lambda_{N}$ (noise, if $\geq 2$ devices transmit), or a message $m \in \mathcal{M}$ (if one device transmits).

No-CD. Transmitters receive no signal. Listeners receive one of two signals: $\lambda_{S}$ (zero or $\geq 2$ devices transmit) or a message $m \in \mathcal{M}$.

Each of the four models comes in both randomized and deterministic variants. A key issue is breaking symmetry. Whereas randomized models easily accomplish this by having devices flip independent random coins, deterministic models depend on having pre-assigned unique IDs to break symmetry.

Randomized Model. In the randomized model, all $n$ devices begin in exactly the same state and can break symmetry by generating private random bits. The number $n$ is unknown and unbounded. The maximum allowed failure probability of a randomized algorithm is at most $1 / \operatorname{poly}(n)$. In a failed execution, devices may consume unbounded energy and never halt $[8,28,29]$.

Deterministic Model. All $n$ devices have unique IDs in the range $[N] \stackrel{\text { def }}{=}\{1, \ldots, N\}$, where $N$ is common knowledge but $n \leq N$ is unknown.

To avoid impossibilities, in the No-CD model it is promised that $n \geq 2$. See Section 3.4 for a discussion of loneliness detection. 
Table 1. Time-energy Tradeoff of Randomized Approximate Counting and Leader Election

\begin{tabular}{|l|c|c|}
\hline & \multicolumn{2}{|c|}{ ENERGY COMPLEXITY } \\
Time COMPLEXITy & Strong-CD or Receiver-CD & Sender-CD or No-CD \\
\hline$O\left(n^{o(1)}\right)$ & $O\left(\log \left(\log { }^{*} n\right)\right)$ & $O\left(\log ^{*} n\right)$ \\
\hline$O\left(\log ^{2+\epsilon} n\right), 0<\epsilon \leq O(1)$ & $O\left(\log \left(\epsilon^{-1} \log \log \log n\right)\right)$ & $O\left(\epsilon^{-1} \log \log \log n\right)$ \\
\hline$O\left(\log ^{2} n\right)$ & $O(\log \log \log n)$ & $O(\log \log n)$ \\
\hline
\end{tabular}

Notice that the Third Line is a Special Case of the Second Line When $\epsilon=1 / \log \log n$.

It could be argued that real-world devices rarely endow transmitters with more collision detection power than receivers, so the Sender-CD model does not merit study. We feel this thinking gets the order backwards. There is a certain cost for equipping tiny devices with extra capabilities (e.g., generating random bits or detecting collisions) so how are we to tell whether adding these capabilities is worth the expense? To answer that question, we first need to determine the complexity of the problems that will ultimately be solved by the network. The goal of this work is to understand the power of various abstract models, not to cleave closely to existing real-world technologies simply because they exist. In this article, we consider the following three fundamental distributed problems:

Leader Election. Exactly one device designates itself the leader and all others designate themselves follower. For technical reasons, we require that the computation ends when the leader sends a message while every follower listens to the channel.

Approximate Counting. At the end of the computation, all devices agree on an estimate $\tilde{n}$ of the network size $n$ such that $\tilde{n}=\Theta(n)$.

Census. At the end of the computation, some device announces a list of the IDs of all devices. We only study this problem in the deterministic model.

Notice that any deterministic algorithm that solves Census is also capable of solving Leader Election and Approximate Counting with the same runtime and energy cost.

\subsection{New Results}

In the randomized model, we show that the energy complexity of Leader Election and Approximate Counting are $\Theta\left(\log ^{*} n\right)$ in Sender-CD and No-CD but $\Theta\left(\log \left(\log ^{*} n\right)\right)$ in Strong-CD and Receiver$\mathrm{CD}$. The lower bounds also apply to the contention resolution problem, and this establishes that the recent $O\left(\log \left(\log ^{*} n\right)\right)$ contention resolution protocol of Bender, Kopelowitz, Pettie, and Young [7] is optimal. Our upper bounds offer a time-energy tradeoff. See Table 1 for the energy cost of our algorithm under different runtime constraints.

For Leader Election, we establish matching bounds in all the deterministic models. In Strong$\mathrm{CD}$ and Sender-CD, Leader Election requires $\Omega(\log \log N)$ energy even when $n=2$, and Census can be solved with $O(\log \log N)$ energy and $O(N)$ time, for any $n \leq N$. However, in No-CD and Receiver-CD, the energy complexity of these problems jumps to $\Theta(\log N)$ [29].

Finally, we prove that when the input is dense in the ID space, meaning $n=\Theta(N)$, Census can actually be computed with only $O(\alpha(N))$ energy and $O(N)$ time, even in No-CD. To our knowledge, this is the first time inverse-Ackermann-type recursion has appeared in distributed computing.

\subsection{Prior Work}

Jurdzinski et al. [27] studied the deterministic energy complexity of Leader Election in the SenderCD model. They proved that dense instances $n=\Theta(N)$ can be solved with $O\left(\log ^{*} N\right)$ energy and claimed that the complexity of the sparse instances is between $\Omega(\log \log N / \log \log \log N)$ and 
$O\left(\log ^{\epsilon} N\right)$. While the lower bound is correct, the algorithm presented in Reference [27] is not. ${ }^{1}$ The most efficient published algorithm uses $O(\sqrt{\log N})$ energy, also due to Jurdzinski et al. [30]. The same authors [28] gave a reduction from randomized Sender-CD Approximate Counting to deterministic Leader Election over ID space $N=O(\log n)$, which, using Reference [30], leads to an $O(\sqrt{\log \log n})$ energy algorithm for Approximate Counting. In Reference [29], the authors gave a method for simulating Sender-CD protocols in the No-CD model and proved that deterministic No-CD Leader Election takes $\Omega(\log N)$ energy. Nakano and Olariu [40] showed that $n$ devices in the Sender-CD model can assign themselves distinct IDs in $\{1, \ldots, n\}$ with $O(\log \log n)$ energy in expectation.

Recently, Bender et al. [7] gave a method for circuit simulation in the Strong-CD model, which led to randomized Approximate Counting and Leader Election protocols using $O\left(\log \left(\log ^{*} n\right)\right)$ energy and $n^{o(1)}$ time. An earlier algorithm of Kardas et al. [32] solves Leader Election in the Strong$\mathrm{CD}$ model in $O\left(\log ^{\epsilon} n\right)$ time using $O(\log \log \log n)$ energy, in expectation but not with high probability.

Most of the previous work in the radio network model has been concerned with time, not energy. Willard [46] proved that $O(\log \log n)$ time is necessary and sufficient for one device to successfully transmit in the Strong-CD model with constant probability; see Reference [41] for tradeoffs between time and success probability. In the Sender-CD model, this problem requires $\Theta\left(\log ^{2} n\right)$ time to solve, with probability $1-1 / \operatorname{poly}(n)[20,31,42]$. Greenberg and Winograd [26] proved that if all devices need to send a message, $\Theta\left(n \log _{n}(N)\right)$ time is necessary and sufficient in the deterministic Strong-CD model.

In multi-hop radio networks, Leader Election and its related problems (e.g., broadcasting and gossiping) have been studied extensively, where the bounds typically depend on both the diameter and size of the network, whether it is directed, and whether randomization and collision detection are available. See, e.g., References [1-3, 9, 12, 13, 15-17, 21, 35-37]. Schneider and Watterhofer [44] investigated the use of collision detection in multihop radio networks when solving archetypal problems such as MIS, $(\Delta+1)$-coloring, and broadcast. Their results showed that the value of collision detection depends on the problem being solved.

Cornejo and Kuhn [14] introduced the beeping model, where no messages are sent; the only signals are $\lambda_{N}$ and $\lambda_{S}$ : noise and silence. The complexity of Approximate Counting was studied in Reference [8] and the "state complexity" of Leader Election was studied in Reference [25].

In adversarial settings, a jammer can interfere with communication. See References $[18,38]$ for leader election protocols resilient to jamming. In a resource-competitive protocol [6], the energy cost of the devices is some function of the energy cost of the jammer. See Reference [5] for resourcecompetitive contention resolution and References [24,34] for resource-competitive point-to-point communication and broadcast protocols.

\subsection{Organization and Technical Overview}

To establish the two sharp exponential separations, we need eight distinct upper and lower bounds. The $O(\log N)$ upper bound on deterministic No-CD Leader Election is trivial, and the matching lower bound in Receiver-CD is provided in Reference [29]. The $O\left(\log \left(\log ^{*} n\right)\right)$ upper bound from Reference [7] on randomized Leader Election and Approximate Counting works only in Strong$\mathrm{CD}$. This article contains proofs of all remaining upper and lower bounds. In addition, we offer a simpler proof of the $\Omega(\log N)$ lower bound in deterministic Receiver-CD and provide an $O(\alpha(N))$ energy protocol for Census in deterministic No-CD when $n=\Theta(N)$.

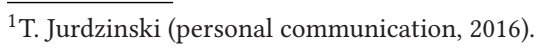


Lower Bounds. In Section 2, we begin with a surprisingly simple proof that protocols solving any non-trivial problem in the deterministic Strong-CD model require $\Omega(\log \log N)$ energy if the devices are adaptive and $\Omega(\log N)$ if they are non-adaptive. It turns out that Receiver-CD algorithms are essentially forced to be non-adaptive, so this yields $\Omega(\log N)$ lower bounds for deterministic Leader Election in Receiver-CD. The $\Omega(\log \log N)$ lower bound combines a decision tree representation of the algorithm with the encoding argument that Katona and Szemerédi [33] used to solve the biclique covering problem of Erdős et al. [19].

In Section 3, we prove the $\Omega\left(\log ^{*} n\right)$ and $\Omega\left(\log \left(\log ^{*} n\right)\right)$ lower bounds on randomized Approximate Counting and Leader Election. These lower bounds begin by embedding any algorithm into an infinite universal DAG that is basically a decision tree with some reconvergent paths. The proof is information theoretic. There are only two methods for devices in Strong-CD and Receiver-CD to learn new information. The first method is via direct communication (in which one device successfully transmits a message and some subset of devices listen); the second method is via inference (in which transmitting or listening devices detect a collision or silence, which informs their future decisions). The information theoretic capacity of the first method is essentially unbounded, whereas the second method is bounded by 1-bit per unit energy in Strong-CD and usually less in Receiver-CD. We show that any algorithm with a reasonable time bound can be forced to learn an approximation of $n$ via the information theoretically well-behaved second method.

Upper Bounds. In Sections 4 and 5, we present all deterministic upper bounds: an $O(\log \log N)$ energy protocol for Census and an $O(\alpha(N))$ energy protocol for dense Census, when $n=\Theta(N)$. Notice that a protocol for Census also solves Leader Election. The first protocol combines van Emde Boas-like recursion with a technique that lets a group of devices function as one device and thereby share energy costs.

In Section 6, we present upper bounds on randomized Leader Election and Approximate Counting. When time is not too constrained, the Sender-CD and Receiver-CD protocols have energy complexity $O\left(\log ^{*} n\right)$ and $O\left(\log \left(\log ^{*} n\right)\right)$. Our protocols naturally adapt to any time bound that is $\Omega\left(\log ^{2} n\right)$, where the energy complexity gradually increases as we approach this lower limit. See Table 1. These protocols are randomized and so do not assume distinct IDs; nonetheless, they use the deterministic $\alpha(N)$ dense Census algorithm of Section 5 .

\section{DETERMINISTIC LOWER BOUNDS}

In this section, we prove deterministic lower bounds for the Successful Communication problem, which immediately leads to the same lower bounds for Leader Election. The goal of Successful Communication is to have some time slot where exactly one device transmits a message while at least one other device listens to the channel. Once a successful communication occurs, the algorithm is terminated on all devices. Throughout the section, we focus on the special case of $n=2$. Each device knows that $n=2$, but not the ID of the other device. In this case, the Strong-CD and Sender-CD models are the same, and the Receiver-CD and No-CD models are the same. Theorem 1 has been proved in Reference [29]; in this section, we offer a simpler proof.

Theorem 1. The deterministic energy complexity of Leader Election is $\Omega(\log N)$ in No-CD and Receiver-CD, even when $n=2$.

Proof. For the case of $n=2$ in No-CD and Receiver-CD, the two devices receive no feedback from the channel until the first successful communication occurs. Thus, to prove the theorem, it suffices to show that the energy cost of any non-adaptive deterministic algorithm $\mathcal{A}$ for Successful Communication is $\Omega(\log N)$. In a non-adaptive algorithm, the sequence of actions taken by a device is solely a function of its ID, not the information it receives from the channel. 


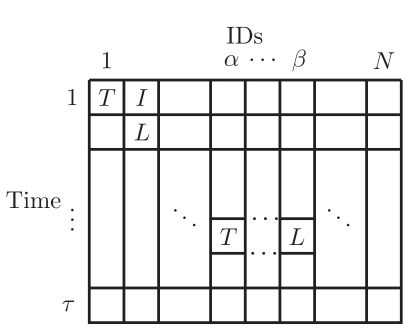

(a)

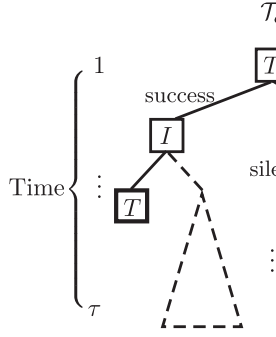

$\mathcal{T}_{\alpha}$

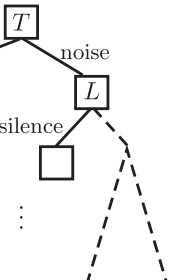

(b)

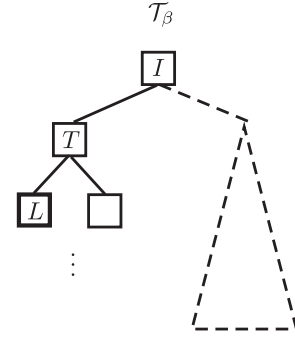

$-1$

Fig. 1. (a) Table for a non-adaptive algorithm. (b) Two binary decision trees $\mathcal{T}_{\alpha}$ and $\mathcal{T}_{\beta}$ for an adaptive algorithm.

Let $\tau=\tau(N)$ be the running time of $\mathcal{A}$. This algorithm can be encoded by a table in the set $\{T, L, I\}^{\tau \times N}$; see Figure 1(a). The $(j, i)$-entry of the table is the action $T$ (transmit), $L$ (listen), or $I$ (idle) taken by the device of ID $i$ at time $j$. Let $E_{i}$ be the energy cost of device of ID $i$, which equals the number of $T$ and $L$ entries in the $i$ th column.

We now prove that $\max _{i} E_{i} \geq \log N$. The proof is inspired by Katona and Szemerédi's [33] lower bound of the biclique covering problem. Encode the $i$ th column by a binary string of length $\tau$ by replacing $T$ with $0, L$ with 1 , and $I$ with either 0 or 1 . There are $2^{\tau-E_{i}}$ possible encodings for column $i$. To solve Successful Communication, the two devices in the network must successfully communicate at some time slot. Thus, for any two distinct IDs $\{\alpha, \beta\}$, there must be a row $r$ such that the $(r, \alpha)$ - and $(r, \beta)$-entries of the table contain one $T$ and one $L$. Therefore, no binary string is an eligible encoding of two distinct columns. Since there are $2^{\tau}$ possible encodings, we have:

$$
\sum_{i=1}^{N} 2^{\tau-E_{i}} \leq 2^{\tau}, \quad \text { which implies } \sum_{i=1}^{N} \frac{1}{2^{E_{i}}} \leq 1 .
$$

This implies $\max _{i} E_{i} \geq \log N$. Moreover, the convexity of $f(x)=2^{-x}$ implies $N \cdot 2^{-\sum_{i=1}^{N} E_{i} / N} \leq 1$, and so $\sum_{i} E_{i} \geq N \log N$. Thus, even on average the energy cost of $\mathcal{A}$ is $\Omega(\log N)$.

Theorem 2. The deterministic energy complexity of Leader Election is $\Omega(\log \log N)$ in Strong-CD and Sender-CD, even when $n=2$.

Proof. It suffices to show that the energy cost of any deterministic algorithm for Successful Communication is $\Omega(\log \log N)$. Suppose we have an algorithm $\mathcal{A}$ for Successful Communication running in $\tau$ time when $n=2$. We represent the behavior of the algorithm on the device with ID $i$ as a binary decision tree $\mathcal{T}_{i}$. Each node in $\mathcal{T}_{i}$ is labeled by $T$ (transmit), $L$ (listen), or $I$ (idle). An $I$-node has one left child and no right child; a $T$-node has two children-a left one indicating collision-free transmission and a right one indicating a collision; an $L$-node has two children-a left one indicating silence and a right one indicating that the device receives a message. Notice that the algorithm terminates once a device reaches the right child of an $L$-node in the decision tree.

The left-right ordering of children is meaningless but essential to making the following argument work. Suppose that we $\operatorname{run} \mathcal{A}$ on two devices with IDs $\alpha$ and $\beta$. Let $t$ be the first time a successful communication occurs. We claim that the paths in $\mathcal{T}_{\alpha}$ and $\mathcal{T}_{\beta}$ corresponding to the execution of $\mathcal{A}$ have exactly the same sequence of $t-1$ left turns and right turns. At any time slot before $t$ the possible actions performed by $\{\alpha, \beta\}$ are $\{I, I\},\{I, T\},\{I, L\},\{L, L\},\{T, T\}$. In all cases, both $\alpha$ and $\beta$ branch to the left child, except for $\{T, T\}$, where they both branch to the right child. At time $t$, the 
actions of the two devices are $\{T, L\}$, and it is only here that they branch in different directions. See Figure 1(b).

We extend each $\mathcal{T}_{i}$ to a full binary tree of depth $\tau$ by adding dummy nodes. The number of nodes in a full binary tree of depth $\tau$ is $2^{\tau}-1$, and so we encode $\mathcal{T}_{i}$ by a binary string of length $2^{\tau}-1$ by listing the nodes in any fixed order (e.g., pre-order traversal), mapping each $T$-node to $0, L$-node to 1 , and each $I$-node or dummy node to either 0 or 1 . For any two distinct IDs $\{\alpha, \beta\}$, there must be a position in the full binary tree such that the corresponding two nodes in $\mathcal{T}_{\alpha}$ and $\mathcal{T}_{\beta}$ are one $T$-node and one $L$-node. Therefore, no binary string is an eligible encoding of $\mathcal{T}_{\alpha}$ and $\mathcal{T}_{\beta}$. If a device with ID $i$ spends energy $E_{i}$, then the number of $T$-nodes and $L$-nodes in $\mathcal{T}_{i}$ is at most $2^{E_{i}}-1$, and so $\mathcal{T}_{i}$ has at most $2^{\left(2^{\tau}-1\right)-\left(2^{E_{i}}-1\right)}$ possible encodings. Thus,

$$
\sum_{i=1}^{N} 2^{\left(2^{\tau}-1\right)-\left(2^{E_{i}}-1\right)} \leq 2^{2^{\tau}-1}, \quad \text { which implies } \quad \sum_{i=1}^{N} \frac{1}{2^{2^{E_{i}}-1}} \leq 1 .
$$

This implies $\max _{i} E_{i} \geq \log (\log N+1)$. Moreover, the convexity of $f(x)=2^{-\left(2^{x}-1\right)}$ implies $N$. $2^{-\left(2^{\sum_{i=1}^{N} E_{i} / N}-1\right)} \leq 1$, and so $\sum_{i} E_{i} \geq N \log (\log N+1)$. Thus, even on average the energy cost of $\mathcal{A}$ is $\Omega(\log \log N)$.

\section{RANDOMIZED LOWER BOUNDS}

In this section, we prove energy lower bounds of randomized algorithms for Approximate Counting. Since No-CD is strictly weaker than Sender-CD, the $\Omega\left(\log ^{*} n\right)$ lower bound also applies to No-CD. Similarly, the $\Omega\left(\log \left(\log ^{*} n\right)\right)$ lower bound for Strong-CD also applies to Receiver-CD.

TheOREM 3. The energy cost of any polynomial time Approximate Counting algorithm with failure probability $1 / n$ is $\Omega\left(\log ^{*} n\right)$ in the Sender-CD and No-CD models.

Theorem 4. The energy cost of any polynomial time Approximate Counting algorithm with failure probability $1 / n$ is $\Omega\left(\log \left(\log ^{*} n\right)\right)$ in the Strong- $C D$ and Receiver-CD models.

In Section 3.1, we introduce the randomized decision tree, which is the foundation of our lower bound proofs. In Section 3.2, we prove Theorem 3. In Section 3.3, we prove Theorem 4. In Section 3.4, we demonstrate that our lower bounds proofs can be adapted to other problems such as Leader Electionand prove the impossibility of loneliness detection (i.e., distinguishing between $n=1$ and $n>1$ ) in randomized No-CD.

\subsection{Randomized Decision Tree}

The process of a device $s$ interacting with the network at time slot $t$ has two phases. During the first phase (action performing phase), $s$ decides on its action, and if this action is to transmit, then $s$ chooses a message $m \in \mathcal{M}$ and transmits $m$. During the second phase (message receiving phase), if $s$ chose to listen or transmit during the first phase, then $s$ may receive a feedback from the channel that depends on the transmissions occurring at this time slot and the collision detection model. The phases partition the time into layers. We write layer $t$ to denote the time right before the first phase of time slot $t$, and layer $t+0.5$ to denote the time right before the second phase of time slot $t$. The choice of the message space $\mathcal{M}$ is irrelevant to our lower bound proof. The cardinality of $\mathcal{M}$ may be finite or infinite.

For a device $s$, the state of $s$ at layer $t$ includes the ordered list of actions taken by $s$ and feedback received from the channel until layer $t$. There is only one possible state in layer 1 , which is the common initial state of all devices before the execution of an algorithm.

Our lower bounds are proved using a single decision tree $\mathcal{T}$, which has unbounded branching factor if $|\mathcal{M}|$ is unbounded. A special directed acyclic graph (DAG) $\mathcal{G}$ is defined to capture the 
behavior of any randomized algorithm, and then the decision tree $\mathcal{T}$ is constructed by "shortcutting" some paths in $\mathcal{G}$.

$D A G \mathcal{G}$. The nodes in $\mathcal{G}$ represent all possible states of a device during the execution of any algorithm. Similarly, the arcs represent all legal transitions between states during the execution of any algorithm. Therefore, each arc connects only nodes in adjacent layers, and the root of $\mathcal{G}$ is the initial state.

Let $t \in \mathbb{Z}^{+}$. A transition from a state $u$ in layer $t$ to a state $v$ in layer $t+0.5$ corresponds to one of the possible $|\mathcal{M}|+2$ actions that can be performed in the first phase of time slot $t$ (i.e., transmit $m$ for some $m \in \mathcal{M}$, listen, or idle). The transitions from a state $u$ in layer $t+0.5$ to a state $v$ in layer $t+1$ are more involved. Based on the action performed in the first phase of time slot $t$ that leads to the state $u$, there are three cases:

- Case: the action is idle. The state $u$ has one outgoing arc corresponding to doing nothing.

- Case: the action is listen. The state $u$ has $|\mathcal{M}|+2$ outgoing arcs in Strong-CD, or $|\mathcal{M}|+1$ in Sender-CD, corresponding to all possible channel feedbacks that can be heard.

- Case: the action is transmit. The state $u$ has two outgoing arcs. The first (resp., second) outgoing arc corresponds to the message transmission succeeding (resp., failing). If a failure took place, then no other device knows which message was sent by the device, and so the content of this message is irrelevant. Thus, all states $u$ in layer $t+0.5$ that correspond to the action transmit and share the same parent have the same child node in layer $t+1$ corresponding to a failure in transmitting the message. The arcs corresponding to failed transmissions are what makes $\mathcal{G}$ a DAG rather than a tree.

Embedding an Algorithm. Any algorithm $\mathcal{A}$ can be embedded into $\mathcal{G}$, as follows: First, appropriate states, depending on $\mathcal{A}$, are designated as terminal states. Without loss of generality, we require that any terminal state must be in layer $t$ for some $t \in \mathbb{Z}^{+}$. Each terminal state is labelled with a specific output for the problem at hand. A device entering a terminal state $u$ terminates with the output associated with the state $u$. Any randomized algorithm is completely described by designating the terminal states together with their outputs and specifying the transition probabilities from states in layer $t$ to states in layer $t+0.5$ for all $t \in \mathbb{Z}^{+}$.

Randomized Decision Tree $\mathcal{T}$. The tree $\mathcal{T}$ is derived from $\mathcal{G}$ as follows: The set of nodes of $\mathcal{T}$ is the set of nodes in $\mathcal{G}$ that are in layer $t$ for some $t \in \mathbb{Z}^{+}$. For any two states $u$ in layer $t \in \mathbb{Z}^{+}$ and $v$ in layer $t+1$ that are linked by a directed path in $\mathcal{G}$, there is a transition from $u$ to $v$ in $\mathcal{T}$. It is straightforward to see that $\mathcal{T}$ is a rooted tree. See Figure 2 for an illustration of both $\mathcal{G}$ and $\mathcal{T}$ in the Strong-CD model with $\mathcal{M}=\left\{m_{1}, \ldots, m_{k}\right\}$. Notice that in the Strong-CD model, a device transmitting a message $m_{i}$ to the channel at a time slot must not hear $\lambda_{S}$ in the same time slot. If the transmission is successful, it hears the message $m_{i}$; otherwise, it hears $\lambda_{N}$.

For a state $u$ in layer $t \in \mathbb{Z}^{+}$, and for an action $x \in\{$ idle, listen, transmit $\}$, we write $p_{u \rightsquigarrow x}$ to denote the probability that a device in state $u$ performs action $x$ in the first phase of time slot $t$.

Time and Energy Complexity. An execution of an algorithm for a device is completely described by a directed path $P=\left(u_{1}, u_{2}, \ldots, u_{k}\right)$ in $\mathcal{T}$ such that $u_{t}$ is in time slot $t$ for each $1 \leq t \leq k$, and $u_{k}$ is the only terminal state in $P$. The runtime of the device is $k$. The amount of energy the device spends is the number of transitions corresponding to listen or transmit in $P$. The time (resp., energy) of an execution of an algorithm is the maximum time (resp., energy) spent by any device.

\subsection{Lower Bound in the Sender-CD Model}

In this section, we prove Theorem 3. Let $\mathcal{A}$ be any $T(n)$ time algorithm for Approximate Counting in Sender-CD with failure probability at most $1 / n$. We show that the energy cost of $\mathcal{A}$ is $\Omega\left(\log ^{*} n\right)$. 


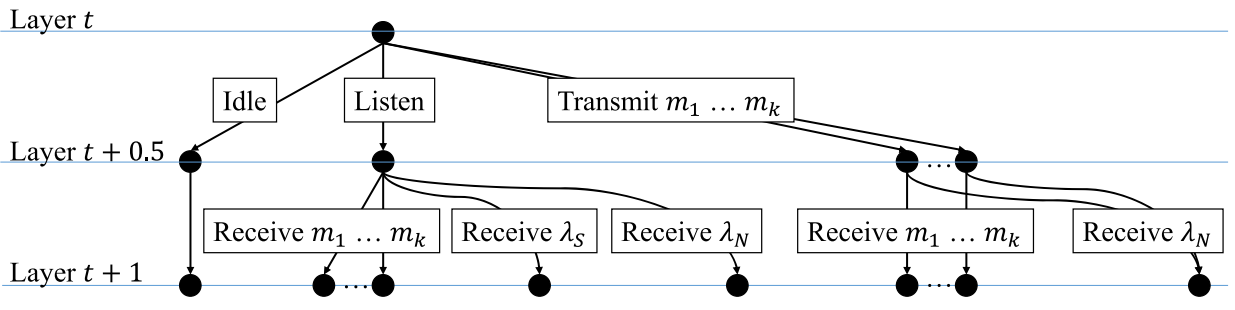

Layer $t$

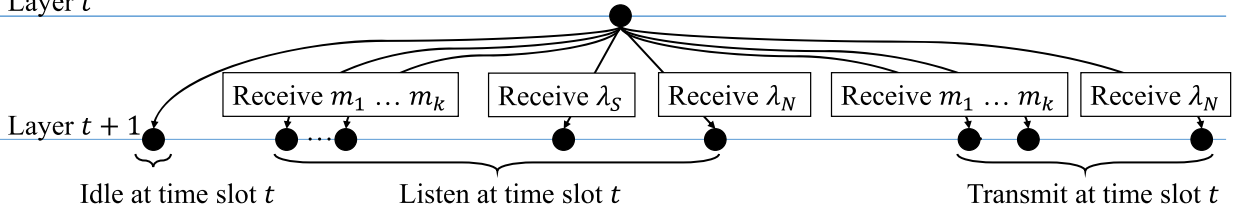

Fig. 2. Upper: a portion of $\mathcal{G}$. Lower: the corresponding portion in $\mathcal{T}$.

Overview. The high-level idea of the proof is as follows: We will carefully select a sequence of network sizes $\left\{n_{i}\right\}$ with checkpoints $\left\{d_{i}\right\}$ such that $d_{i}<n_{i}<d_{i+1}$ and $T\left(n_{i}\right)<d_{i+1}$. There are two main components in the proof. The first component is to demonstrate that, with probability $1-$ $1 /$ poly $\left(n_{i}\right)$, no message is successfully transmitted before time $d_{i}$ when running $\mathcal{A}$ on $n_{i}$ devices, i.e., every transmission ends in a collision. This limits the amount of information that could be learned from a device. The second component is to prove that, for $j>i$, in order for a device $s$ to learn enough information to distinguish between $n_{i}$ and $n_{j}$ within $T\left(n_{i}\right)<d_{i+1}$ time slots, the device $s$ must use at least one unit of energy within time interval $\left[d_{i}, d_{i+1}-1\right]$. The intuition is briefly explained as follows: Given that $n \in\left\{n_{i}, n_{j}\right\}$, with high probability, every transmission ends in a collision before time $d_{i}$, and so $s$ has not yet obtained enough information to distinguish between $n_{i}$ and $n_{j}$ by the time $d_{i}-1$. The only way $s$ can gain information is to use energy, i.e., listen or transmit. It is required that $s$ terminates by time $T\left(n_{i}\right)$ if the total number of devices is $n_{i}$, and so $s$ must use at least one unit of energy within time interval $\left[d_{i}, T\left(n_{i}\right)\right] \subseteq\left[d_{i}, d_{i+1}-1\right]$.

Truncated Decision Tree. The no-communication tree $\mathcal{T}_{\text {no-comm }}$ is defined as the subtree of $\mathcal{T}$ induced by the set of all states $u$ such that no transition in the path from the root to $u$ corresponds to receiving a message in $\mathcal{M}$. In other words, $\mathcal{T}_{\text {no-comm }}$ contains exactly the states whose execution history contains no successful communication. Notice that in Sender-CD each state in $\mathcal{T}_{\text {no-comm }}$ has exactly three children, and the three children correspond to the following three pairs of action performed and channel feedback received: (transmit, $\lambda_{S}$ ), (listen, $\lambda_{S}$ ), and (idle, N/A).

For each state $u$ at layer $t$ of the tree $\mathcal{T}_{\text {no-comm }}$, we define the probability estimate $p_{u}$ inductively as follows: If $u$ is the root, $p_{u}=1$; otherwise, $p_{u}=p_{v} \cdot p_{v \rightsquigarrow x}$, where $v$ is the parent of $u$, and $x$ is the action performed at time slot $t-1$ that leads to the state $u$. Recall that $p_{v \rightsquigarrow x}$ is defined as the probability for a device in $v$ (which is a state in layer $t-1$ ) to perform $x$ at time slot $t-1$. Intuitively, if no message is successfully sent in an execution of $\mathcal{A}$, the proportion of devices entering $u$ is well concentrated around $p_{u}$, given that $p_{u}$ is high enough. See Figure 3 for an illustration of no-communication tree $\mathcal{T}_{\text {no-comm }}$ and probability estimates in the Sender-CD model.

Given the runtime constraint $T(n)$ for $\mathcal{A}$, we select an infinite sequence of checkpoints as follows: $d_{1}$ is a sufficiently large constant to meet the requirements in the subsequent analysis; for each $i>1, d_{i}$ is any number satisfying the two criteria (i) $d_{i} \geq 2^{2^{2^{2^{d_{i-1}}}}}$ and (ii) $d_{i} \geq T\left(n^{\prime}\right)+1$ for all 

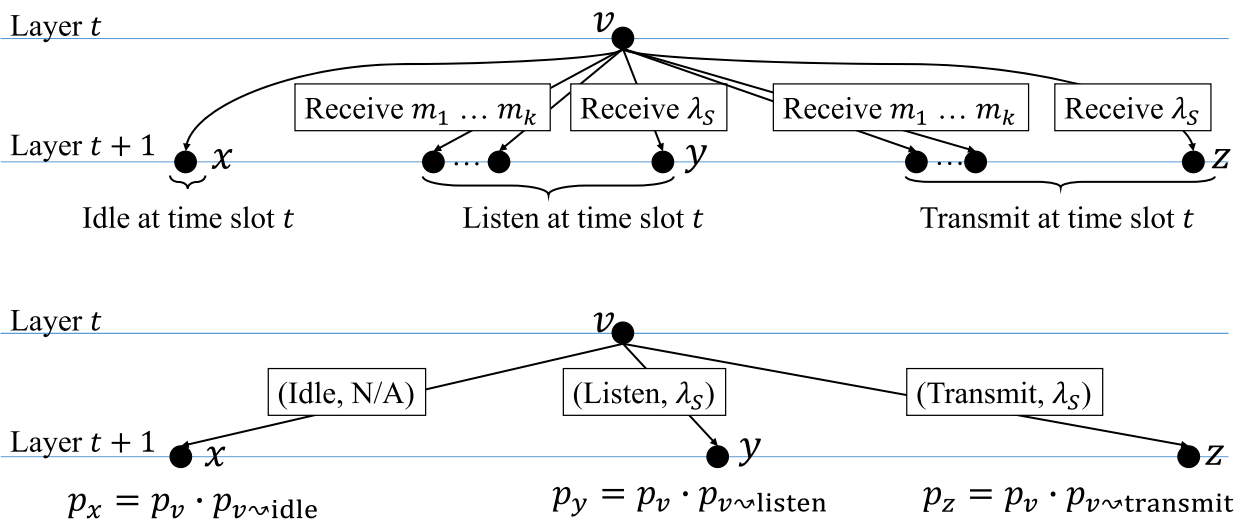

Fig. 3. Upper: a portion of the tree $\mathcal{T}$ in Sender-CD. Lower: the corresponding portion in the nocommunication tree $\mathcal{T}_{\text {no-comm. }}$.

$2^{2^{d_{i-1}}}<n^{\prime}<2^{2^{2^{2^{d_{i-1}}}}}$. For example, if $T(n)$ is a non-decreasing function and $T(n) \geq n$, then we can simply set $d_{i}=T\left(2^{2^{2^{d^{d_{i-1}}}}}\right)+1 .^{2}$

LEMma 1. For each index $i$, there exists a number $n_{i}$ such that $2^{2^{d_{i}}}<n_{i}<2^{2^{2^{2^{d_{i}}}}}$ and for each state $u \in \mathcal{T}_{\text {no-comm }}$ at layer at most $d_{i}$, either $p_{u} \leq n_{i}^{-10}$ or $p_{u} \geq n_{i}^{-1 / 10}$.

Proof. Define $m_{1}=2^{2^{d_{i}}}+1$; for $1<k \leq 3^{d_{i}}$, define $m_{k}=m_{k-1}^{100}+1$. It is straightforward to see that $2^{2^{d_{i}}}<m_{1}<m_{2}<\cdots<m_{3} d_{i}<2^{2^{2^{d_{i}}}}$, so long as $d_{i}$ is greater than some universal constant. For each state $u \in \mathcal{T}_{\text {no-comm }}$ at layer at most $d_{i}$, there exists at most one $m_{k}$ with $m_{k}{ }^{-10}<p_{u}<$ $m_{k}{ }^{-1 / 10}$. Recall that $\mathcal{T}_{\text {no-comm }}$ has branching factor 3 , and hence the number of states up to layer $d_{i}$ is less than $3^{d_{i}}$. By the pigeonhole principle, among the $3^{d_{i}}$ distinct integers $m_{1}, m_{2}, \ldots, m_{3} d_{i}$, there exists one integer $n_{i}$ such that, for each state $u \in \mathcal{T}_{\text {no-comm }}$ at layer at most $d_{i}$, either $p_{u} \leq n_{i}^{-10}$ or $p_{u} \geq n_{i}^{-1 / 10}$.

For each index $i$, the parameter $n_{i}$ is chosen to meet the statement of Lemma 1. Recall that the goal of $\mathcal{A}$ is to calculate an estimate $\tilde{n}$ that is within a multiplicative factor $c$ of $n$, where $c>1$ is some constant. We select the first checkpoint $d_{1}$ to be a large enough constant such that $c \cdot n_{i}<n_{i+1} / c$ for all $i$. We define $\mathcal{T}_{i}$ as the subtree of $\mathcal{T}_{\text {no-comm }}$ that consists of all states $u$ up to layer $d_{i}$ such that $p_{u} \geq n_{i}^{-1 / 10}$. Notice that $\mathcal{T}_{i} \subseteq \mathcal{T}_{i+1}$ for all $i$.

Consider an execution of $\mathcal{A}$ on $n_{i}$ devices. Let $t \in\left[1, d_{i}\right]$, and denote $\mathcal{P}_{i}^{(t)}$ as the event that, for each state $u$ in layer $t$ of the decision tree $\mathcal{T}$, the number of devices entering $u$ is within the range $n_{i} \cdot p_{u} \pm(t-1) \cdot n_{i}^{0.6}$ if $u$ is in layer $t$ of $\mathcal{T}_{i}$, and is 0 if $u \notin \mathcal{T}_{i}$. We write $\mathcal{P}_{i}=\bigcap_{t=1}^{d_{i}} \mathcal{P}_{i}^{(t)}$. Notice that $\mathcal{P}_{i}^{(1)}$ holds with probability 1 .

LEMma 2. Let $t<d_{i}$ and $x \in\{$ transmit, listen, idle $\}$. Let $v$ be a state in layer $t$ of $\mathcal{T}_{i}$ such that the number of devices entering $v$ is within $n_{i} \cdot p_{v} \pm(t-1) \cdot n_{i}^{0.6}$. Define $m$ as the number of devices that are in state $v$ and perform action $x$ at time $t$. The following holds with probability $1-O\left(n_{i}^{-9}\right)$. If $p_{v} \cdot p_{v \rightsquigarrow x} \geq n_{i}^{-1 / 10}$, then $m$ is within $n_{i} \cdot p_{v} \cdot p_{v \rightsquigarrow x} \pm t \cdot n_{i}^{0.6} ;$ if $p_{v} \cdot p_{v \rightsquigarrow x} \leq n_{i}^{-10}$, then $m=0$.

\footnotetext{
${ }^{2}$ It might be possible to reduce the height of the power tower. However, having any constant height is sufficient to prove the desired lower bound, so we do not need to optimize the constant.
} 
Proof. According to our choice of $n_{i}$, for each state $u \in \mathcal{T}_{\text {no-comm }}$ at layer at most $d_{i}$, either $p_{u} \leq n_{i}^{-10}$ or $p_{u} \geq n_{i}^{-1 / 10}$. Since $p_{v} \cdot p_{v \rightsquigarrow x}=p_{u}$ for some $u \in \mathcal{T}_{\text {no-comm }}$ at layer at most $d_{i}$, either (i) $n_{i}^{-10} \geq p_{v} \cdot p_{v \rightsquigarrow x}$ or (ii) $p_{v} \cdot p_{v \rightsquigarrow x} \geq n_{i}^{-1 / 10}$ is true.

First, consider the case $n_{i}^{-10} \geq p_{v} \cdot p_{v \rightsquigarrow x}$. Notice that $p_{v \rightsquigarrow x} \leq n_{i}^{-10} / p_{v} \leq n_{i}^{-9.9}$, and recall $t \leq$ $d_{i}<\log \log n_{i}$. We upper bound the expected value of $m$ as follows.

$$
\begin{aligned}
\mathrm{E}[m] & \leq\left(n_{i} \cdot p_{v}+(t-1) \cdot n_{i}^{0.6}\right) \cdot p_{v \rightsquigarrow x} \\
& \leq n_{i}^{-9}+(t-1) \cdot n_{i}^{0.6} \cdot p_{v \rightsquigarrow x} \\
& \leq n_{i}^{-9}+(t-1) \cdot n_{i}^{-9.3} \\
& <2 n_{i}^{-9} .
\end{aligned}
$$

By Markov's inequality, $m=0$ with probability at least $1-2 n_{i}^{-9}$, as desired.

Next, consider the case $p_{v} \cdot p_{v \rightsquigarrow x} \geq n_{i}^{-1 / 10}$. The value of $\mathrm{E}[m]$ is within $p_{v \rightsquigarrow x} \cdot\left(n_{i} \cdot p_{v} \pm(t-\right.$ 1) $\left.\cdot n_{i}^{0.6}\right)$, which is within the range $n_{i} \cdot p_{v} \cdot p_{v \rightsquigarrow x} \pm(t-1) \cdot n_{i}^{0.6}$. Let $\delta=n_{i}^{-0.4} / 2$. A simple calculation shows that $\delta \cdot \mathrm{E}[m]<n_{i}^{0.6}$ and $\mathrm{E}[m]>n_{i}^{0.9} / 2$. Notice that each device in the state $v$ decides which action to perform next independently. By a Chernoff bound, the probability that $m$ is within $1 \pm \delta$ factor of $\mathrm{E}[m]$ is at least $1-2 \exp \left(-\delta^{2} \cdot \mathrm{E}[m] / 3\right) \geq 1-2 \exp \left(-\left(n_{i}^{-0.4} / 2\right)^{2} \cdot\left(n_{i}^{0.9} / 2\right) / 3\right)>1-$ $O\left(n_{i}^{-9}\right)$. Therefore, with such probability, $m$ is in the range $\mathrm{E}[m](1 \pm \delta)$, which is within $n_{i} \cdot p_{v} \cdot$ $p_{v \rightsquigarrow x} \pm t \cdot n_{i}^{0.6}$, as desired.

Lemma 3. For an execution of $\mathcal{A}$ on $n_{i}$ devices, $\mathcal{P}_{i}$ holds with probability at least $1-n_{i}^{-7}$.

Proof. For the base case, $\mathcal{P}_{i}^{(1)}$ holds with probability 1 . For each $1<t \leq d_{i}$, we will show that $\operatorname{Pr}\left[\mathcal{P}_{i}^{(t)} \mid \mathcal{P}_{i}^{(t-1)}\right]=1-O\left(n_{i}^{-8}\right)$. Therefore, by a union bound on all $t \in\left\{1, \ldots, d_{i}\right\}$, we have:

$$
\operatorname{Pr}\left[\mathcal{P}_{i}\right]=\operatorname{Pr}\left[\bigcap_{t=1}^{d_{i}} \mathcal{P}_{i}^{(t)}\right] \geq 1-d_{i} \cdot O\left(n_{i}^{-8}\right) \geq 1-O\left(n_{i}^{-8} \log \log n_{i}\right) \geq 1-n_{i}^{-7} .
$$

Let $1<t \leq d_{i}$. Suppose that $\mathcal{P}_{i}^{(t-1)}$ holds. This implies that, for each state $v$ in layer $t-1$ of $\mathcal{T}_{i}$, the number of devices entering $v$ is within $n_{i} \cdot p_{v} \pm(t-2) \cdot n_{i}^{0.6}$. The statement of Lemma 2 holds for at most $3^{t-1}$ choices of states $v$ in layer $t-1$ of $\mathcal{T}_{i}$, and all 3 choices of $x \in$ \{transmit, listen, idle $\}$, with probability at least

$$
1-3 \cdot 3^{t-1} \cdot O\left(n_{i}^{-9}\right) \geq 1-O\left(n_{i}^{-9} \text { poly } \log n_{i}\right) \geq 1-O\left(n_{i}^{-8}\right) .
$$

In particular, this implies that, with probability $1-O\left(n_{i}^{-8}\right)$, at time $t-1$, the number of devices transmitting is either 0 or greater than 1 , which implies that no message is successfully sent. Therefore, at layer $t$, all devices are confined in states within $\mathcal{T}_{\text {no-comm }}$. Let $u$ be the child of $v$ in $\mathcal{T}_{\text {no-comm }}$ such that the arc $(v, u)$ corresponds to action $x$. Due to our choices of $n_{i}$ and $\mathcal{T}_{i}$, if $u \in \mathcal{T}_{i}$, then $p_{u}=p_{v} \cdot p_{v \rightsquigarrow x} \geq n_{i}^{-1 / 10}$; if $u \notin \mathcal{T}_{i}$, then $p_{u}=p_{v} \cdot p_{v \rightsquigarrow x} \leq n_{i}^{-10}$. Therefore, in view of the statement of Lemma $2, \mathcal{P}_{i}^{(t)}$ holds with probability at least $1-O\left(n_{i}^{-8}\right)$.

Lemma 4. The no-communication tree $\mathcal{T}_{\text {no-comm }}$ has no terminal state $u$ with $p_{u} \neq 0$.

Proof. Suppose that $u \in \mathcal{T}_{\text {no-comm }}$ is a terminal state with $p_{u} \neq 0$. Then there exists an index $i$ such that for all $j \geq i, u \in \mathcal{T}_{j}$. Among all $\left\{n_{j}\right\}_{j \geq i}$, the decision of $u$ is a correct estimate of at most one $n_{j}$. Therefore, the adversary can choose one network size $n_{j^{\prime}}$ from $\left\{n_{j}\right\}_{j \geq i}$ such that when $\mathcal{A}$ is executed on $n_{j^{\prime}}$ devices, any device entering $u$ gives a wrong estimate of $n_{j^{\prime}}$. By Lemma 3, with probability $1-n_{j^{\prime}}^{-7}>1 / n_{j^{\prime}}$, there is a device entering $u$, and hence the algorithm fails with probability higher than $1 / n_{j^{\prime}}$, a contradiction. 
In the following lemma, we show how to force energy expenditure of devices.

LEMma 5. Define $p_{\text {idle }}^{(i)}$ as the maximum probability for a device $s$ that is in a state $u$ in layer $d_{i}$ of $\mathcal{T}_{i}$ to be idle throughout the time interval $\left[d_{i}, d_{i+1}-1\right]$, where the maximum ranges over all states in layer $d_{i}$ of $\mathcal{T}_{i}$. Then $p_{\text {idle }}^{(i)}<2^{-d_{i}}$.

Proof. For a device $s$ to terminate within the time constraint $T\left(n_{i}\right)$, the device $s$ must leave the tree $\mathcal{T}_{\text {no-comm }}$ by time $T\left(n_{i}\right)<d_{i+1}$ due to Lemma 4 . Suppose that the device $s$ is currently in a state $u$ in layer $d_{i}$ of $\mathcal{T}_{i} \subseteq \mathcal{T}_{\text {no-comm }}$. To leave the tree $\mathcal{T}_{\text {no-comm }}$ by time $T\left(n_{i}\right)$, the device $s$ must successfully hear some message $m \in \mathcal{M}$ by time $T\left(n_{i}\right)$. Since $\mathcal{T}_{i} \subseteq \mathcal{T}_{\text {no-comm }}, s$ has not heard any message by time $d_{i}-1$, and so at least one unit of energy expenditure in the time interval $\left[d_{i}, d_{i+1}-1\right]$ is required.

Recall that if $\mathcal{P}_{i}$ occurs, then all devices are confined in $\mathcal{T}_{i}$ up to layer $d_{i}$. If we execute $\mathcal{A}$ on $n_{i}$ devices, then the probability that the runtime of a device exceeds $T\left(n_{i}\right)$ is at least $\operatorname{Pr}\left[\mathcal{P}_{i}\right] \cdot p_{\mathrm{idle}}^{(i)}$, and so we must have $1 / n_{i} \geq \operatorname{Pr}\left[\mathcal{P}_{i}\right] \cdot p_{\text {idle }}^{(i)}$. By Lemma 3 , we have $\operatorname{Pr}\left[\mathcal{P}_{i}\right] \geq 1-n_{i}^{-7}>1 / 2$. Therefore, $p_{\text {idle }}^{(i)} \leq 1 /\left(n_{i} \operatorname{Pr}\left[\mathcal{P}_{i}\right]\right)<2 / n_{i} \leq 2 \cdot 2^{-2^{d_{i}}}<2^{-d_{i}}$, as desired.

We are now in a position to prove the main result of this section.

Lemma 6. For any $i \geq 1$, there exists a network size $n$ satisfying $d_{i}<n<d_{i+1}$ such that if $\mathcal{A}$ is executed on $n$ devices, for any device $s$, with probability at least $1 / 2$ the device $s$ spends at least one unit of energy in each of the time intervals $\left[d_{j}, d_{j+1}-1\right], 1 \leq j \leq i$.

Proof. We select $n=n_{i}$. Consider an execution of $\mathcal{A}$ on $n_{i}$ devices, and let $s$ be any one of the $n_{i}$ devices. Let $j \in\{1, \ldots, i\}$. We claim that, given that $\mathcal{P}_{i}$ holds, with probability $1-2 \cdot 2^{-d_{j}}$ the device $s$ spends at least one unit of energy in the interval $\left[d_{j}, d_{j+1}-1\right]$. Then, by a union bound on all $j \in\{1, \ldots, i\}$, the probability that the device $s$ spends at least one unit of energy in each of the intervals $\left[d_{j}, d_{j+1}-1\right], 1 \leq j \leq i$, is at least $1-\left(1-\operatorname{Pr}\left[\mathcal{P}_{i}\right]\right)-2 \sum_{j=1}^{i} 2^{-d_{j}}$, which is greater than $1 / 2$ if $d_{1}$ is chosen as a sufficiently large constant.

Next, we prove the above claim. Suppose $\mathcal{P}_{i}$ holds. In view of Lemma 5 , if $s$ enters a state in layer $d_{j}$ of $\mathcal{T}_{j}$, then $s$ spends at least one unit of energy in the time interval $\left[d_{j}, d_{j+1}-1\right]$ with probability $1-2^{-d_{j}}$. Thus, all we need to do is to show that the probability that $s$ enters a state in layer $d_{j}$ of $\mathcal{T}_{j}$ is at least $1-2^{-d_{j}}$.

Recall that $\mathcal{T}_{j}$ is a subtree of $\mathcal{T}_{i}$. Let $u$ be a state in layer $d_{j}$ that does not belong to $\mathcal{T}_{j}$. We have $p_{u}<n_{j}^{-1 / 10}$. Since $\mathcal{P}_{i}$ holds, the number of devices entering the state $u$ is at most $n_{i} \cdot n_{j}^{-1 / 10}+\left(d_{j}-\right.$ 1) $\cdot n_{i}^{0.6}$. Since there are at most $3^{d_{j}}$ states in layer $d_{j}$ of $\mathcal{T}_{i}$, the proportion of the devices that do not enter a state in layer $d_{j}$ of $\mathcal{T}_{j}$ is at most

$$
\frac{1}{n_{i}}\left(n_{i} \cdot n_{j}^{-1 / 10}+\left(d_{j}-1\right) \cdot n_{i}^{0.6}\right) \cdot 3^{d_{j}}=\left(n_{j}^{-1 / 10}+\left(d_{j}-1\right) \cdot n_{i}^{-0.4}\right) \cdot 3^{d_{j}}<2^{-d_{j}},
$$

since $n_{i} \geq n_{j} \geq 2^{2^{d_{j}}}$.

If it is the case that $T(n) \leq \exp ^{(\ell)}(n)$, for some constant $\ell$, where $\exp ^{(i)}$ is iterated $i$-fold application of exp, then it is possible to set the checkpoints so $\arg \max _{i}\left(d_{i}<n\right)=\Theta\left(\log ^{*} n\right)$, and so Lemma 6 implies that the energy cost $\mathcal{A}$ is $\Omega\left(\log ^{*} n\right)$. Therefore, we conclude Theorem 3 (which is the case of $T(n)=O(\operatorname{poly}(n)))$.

\subsection{Lower Bound in the Strong-CD Model}

In this section, we prove Theorem 4 . Let $\mathcal{A}$ be any $T(n)$ time algorithm for Approximate Counting in Strong-CD with failure probability at most $1 / n$. We show that the energy cost of $\mathcal{A}$ is $\Omega\left(\log \log ^{*} n\right)$. 
Overview. Similar to Section 3.2, we will construct a sequence of network sizes $\left\{n_{i}\right\}$ with checkpoints $\left\{d_{i}\right\}$ such $d_{i}<n_{i}<d_{i+1}$ and $T\left(n_{i}\right)<d_{i+1}$. Each index $i$ is associated with a truncated decision tree $\mathcal{T}_{i}$ such that if we execute $\mathcal{A}$ on $n_{i}$ devices, then the execution history of all devices until time $d_{i}$ are confined to $\mathcal{T}_{i}$ with probability $1-1 / \operatorname{poly}\left(n_{i}\right)$.

Suppose that the actual network size $n$ is chosen from the set $S=\left\{n_{1}, \ldots, n_{k}\right\}$. The proof in Section 3.2 says that it costs $\Omega(k)$ energy to estimate $n$, when $n=n_{k}$. However, in the Strong-CD model, the devices are capable of differentiating between silence and noise, and so they are able to perform a binary search on $S$, which costs only $O(\log k)$ energy to estimate $n$.

The high-level idea of our proof of Theorem 4 is to demonstrate that this binary search strategy is optimal. We will carefully select a path $P$ in $\mathcal{T}_{\text {no-comm }}$ reflecting a worst-case scenario of the binary search, and we will show that the energy consumption of any device whose execution history follows the path $P$ is $\Omega\left(\log \left(\log ^{*} n\right)\right)$.

Basic Setup. The definitions of the no-communication tree $\mathcal{T}_{\text {no-comm }}$ and probability estimate $p_{u}$ are adapted from Section 3.2. In the Strong-CD model, each state in $\mathcal{T}_{\text {no-comm }}$ has exactly four children, corresponding to all valid combinations of $\left\{\lambda_{S}, \lambda_{N}\right\}$ and $\{$ transmit, listen, idle $\}$ : (transmit, $\lambda_{N}$ ), (listen, $\lambda_{S}$ ), (listen, $\lambda_{N}$ ), and (idle, N/A). Notice that a device transmitting a message never hears silence in the Strong-CD model. The definition of the checkpoints $d_{i}$ and how we select the network sizes $n_{i}$ are also the same as in Section 3.2.

Truncated Subtrees. The subtrees $\left\{\mathcal{T}_{i}\right\}$ are defined differently. For each index $i$, the subtree $\mathcal{T}_{i}$, along with the sequence $\left\{m_{i, t}\right\}_{1 \leq t \leq d_{i}-1}$ indicating a likely status (noise or silence) of the channel at time slot $t$ when $n=n_{i}$, is constructed layer-by-layer as follows:

Base Case. The first layer of $\mathcal{T}_{i}$ consists of only the initial state.

Inductive Step. For each $1<t \leq d_{i}$, suppose that layer $t-1$ of $\mathcal{T}_{i}$ has been defined. If there is at least one state $v$ in layer $t-1$ of $\mathcal{T}_{i}$ with $p_{v} \cdot p_{v \rightsquigarrow \text { transmit }} \geq n_{i}{ }^{-1 / 10}$, set $m_{i, t-1}=\lambda_{N}$; otherwise, set $m_{i, t-1}=\lambda_{S}$. Let $u$ be a state in layer $t$ that is a child of a state $w$ in $\mathcal{T}_{i}$. Let $m \in\left\{\lambda_{N}, \lambda_{S}, \mathrm{~N} / \mathrm{A}\right\}$ and $x \in\{$ transmit, listen, idle $\}$ be the channel feedback associated with the arc $(w, u)$. We add $u$ to layer $t$ of $\mathcal{T}_{i}$ if the following two conditions are met: (i) $p_{u} \geq n_{i}^{-1 / 10}$ and (ii) $x=$ idle or $m=m_{i, t-1}$.

We discuss some properties of $\mathcal{T}_{i}$. All states in $\mathcal{T}_{i}$ are in layers $\left[1, d_{i}\right]$. Let $w$ be a layer $(t-1)$ state in $\mathcal{T}_{i}$, and let $u_{1}$ and $u_{2}$ be the two children of $v$ corresponding to (listen, $\lambda_{S}$ ) and (listen, $\lambda_{N}$ ). Due to the definition of $\mathcal{T}_{i}$, at most one of $u_{1}$ and $u_{2}$ is in $\mathcal{T}_{i}$, and so each state in $\mathcal{T}_{i}$ has at most three children. We do not have $\mathcal{T}_{1} \subseteq \mathcal{T}_{2} \subseteq \cdots$ in general.

We define the event $\mathcal{P}_{i}$ in the same way as in Section 3.2, but using the new definition of $\mathcal{T}_{i}$. We have the following lemma, whose proof is essentially the same as that of Lemma 3 . The only difference is that we need to show that for each time slot $t$, the designated channel feedback $m_{i, t} \in\left\{\lambda_{N}, \lambda_{S}\right\}$ occurs with probability $1-1 / \operatorname{poly}\left(n_{i}\right)$, given that the event $\mathcal{P}_{i}^{(t)}$ occurs; this can be achieved via a proof similar to that of Lemma 2 .

Lemma 7. For an execution of $\mathcal{A}$ on $n_{i}$ devices, $\mathcal{P}_{i}$ holds with probability at least $1-n_{i}^{-7}$.

A difference between Strong-CD and Sender-CD is that in the Strong-CD model it is possible to have terminal states in $\mathcal{T}_{\text {no-comm }}$. However, there is a simple sufficient condition to guarantee that a state $u$ in $\mathcal{T}_{\text {no-comm }}$ is not a terminal state.

LEMmA 8. Let $u$ be any state in both $\mathcal{T}_{i}$ and $\mathcal{T}_{j}$ for some $i \neq j$. Then $u$ is not a terminal state.

Proof. Suppose that $u$ is a terminal state. The decision of $u$ is a correct estimate of at most one of $\left\{n_{i}, n_{j}\right\}$. Without loss of generality, assume that the decision of $u$ is an incorrect estimate 
of $n_{j}$. When $\mathcal{A}$ is executed on $n_{j}$ devices, any device entering $u$ gives a wrong estimate of $n_{j}$. By Lemma 7 , with probability $1-n_{j}^{-7}>1 / n_{j}$, there is a device entering $u$, and hence the algorithm fails with probability higher than $1 / n_{j}$, a contradiction.

Let $k \geq 3$ be an integer. Consider the set $\left\{n_{1}, \ldots, n_{k}\right\}$. Our goal is to find an index $\hat{i}$ such that, during an execution of $\mathcal{A}$ on $n_{\hat{i}}$ devices, with probability $1-1 / \operatorname{poly}\left(n_{\hat{i}}\right)$, there exists a device that uses $\Omega(\log k)$ energy. This is achieved by constructing a high energy path $P=\left(u_{1}, u_{2}, \ldots, u_{\hat{t}}\right)$, along with a sequence of sets of active indices $K_{1} \supseteq K_{2} \supseteq \cdots \supseteq K_{\hat{t}}$ in such a way that $i \in K_{t}$ implies $u_{t} \in \mathcal{T}_{i}$. The path $P$ is a directed path in the tree $\mathcal{T}_{\text {no-comm }}$, and $u_{t}$ belongs to layer $t$, for each $t$. The number $\hat{t}$ will de chosen later. We will later see that any device entering the state $u_{t}$ is unable to distinguish between $\left\{n_{i}\right\}_{i \in K_{t}}$. The path $P$ is selected to contain at least $\Omega(\log k)$ transitions corresponding to listen or transmit. Thus, choosing $\hat{i}$ as any index in $K_{\hat{t}}$ implies $u_{\hat{t}} \in \mathcal{T}_{\hat{i}}$, and so $\hat{t} \leq d_{\hat{i}}$. Then, Lemma 7 and the definition of $\mathcal{P}_{i}$ imply that in an execution of $\mathcal{A}$ on $n_{\hat{i}}$ devices, with probability $1-n_{\hat{i}}^{-7}$, at least $n_{\hat{i}} \cdot p_{u_{\hat{t}}}-(\hat{t}-1) \cdot n_{\hat{i}}^{0.6}=\Omega\left(n_{\hat{i}}^{0.9}\right)>1$ device enters the state $u_{\hat{t}}$ along the path $P$, and any such device uses $\Omega(\log k)$ energy.

One may attempt to construct the path $P$ by a greedy algorithm that iteratively extends the path by choosing the child state $v$ with the highest probability estimate $p_{v}$. The "regular update" in our construction of $P$ is based on this strategy. However, this strategy alone is insufficient to warrant any energy expenditure in $P$.

We briefly discuss how we force energy expenditure. Recall that (i) $i \in K_{t}$ implies $u_{t} \in \mathcal{T}_{i}$, and (ii) any device entering $u_{t}$ is unable to distinguish between the network sizes in $\left\{n_{i}\right\}_{i \in K_{t}}$. Suppose $i \in K_{d_{i}}$, and let $s$ be any device in the state $u_{d_{i}}$. The probability that $s$ remains idle throughout the time interval $\left[d_{i}, d_{i+1}-1\right]$ must be small. The reason is that $s$ needs to learn whether the underlying network size is $n_{i}$ by the time $T\left(n_{i}\right)<d_{i+1}$. Suppose that $\left(u_{1}, \ldots, u_{t}\right)$ have been defined, and we have $t=d_{i}$ and $i \in K_{d_{i}}$. Then it is possible to extend $\left(u_{1}, \ldots, u_{t}\right)$ in such a way that guarantees one energy expenditure in the time interval $\left[d_{i}, d_{i+1}-1\right]$. This corresponds to the "special update" in our construction of $P$.

Construction of the High Energy Path. The path $P=\left(u_{1}, \ldots, u_{\hat{t}}\right)$ and the sequence $K_{1} \supseteq K_{2} \supseteq$ $\cdots \supseteq K_{\hat{t}}$ are defined as follows: We initialize $\tilde{P}=\left(u_{1}\right)$ with $u_{1}$ being the initial state, and let $K_{1}=$ $\{1,2, \ldots, k\}$.

Stopping Criterion. The following update rules are applied repeatedly to extend the current path $\tilde{P}=\left(u_{1}, \ldots, u_{t}\right)$ to a longer path $\left(u_{1}, \ldots, u_{t^{\prime}}\right)$ (for some $\left.t^{\prime}>t\right)$ until the stopping criterion $\left|K_{t}\right|<4$ is reached. Then, we set $P=\tilde{P}$. We will later see in the calculation of the shrinking rate of $\left|K_{t}\right|$ in the proof of Lemma 14 that the stopping criterion implies $\left|K_{t^{\prime \prime}}\right| \geq 2$ for all $1 \leq t^{\prime \prime} \leq \hat{t}$ in the final path $P=\left(u_{1}, \ldots, u_{\hat{t}}\right)$. By Lemma 8 , this implies that all states in $P$ are not terminal states.

Regular Update. We apply this rule if $t \neq d_{i}$ for all $i \in K_{t}$. Let $x^{\star} \in\{$ transmit, listen, idle $\}$ be chosen to maximize $p_{u_{t} \rightsquigarrow x^{\star}}$. If $x^{\star}=$ idle, append the child of $u_{t}$ that corresponds to performing $x^{\star}$ at time $t$ to the end of $\tilde{P}$, and set $K_{t+1}=K_{t}$. In what follows, suppose $x^{\star} \in\{$ transmit, listen $\}$. If $x^{\star}=$ transmit, let $m^{\star}=\lambda_{N}$. If $x^{\star}=$ listen, let $m^{\star} \in\left\{\lambda_{S}, \lambda_{N}\right\}$ be chosen to maximize the number of indices $j \in K_{t}$ with $m_{j, t}=m^{\star}$. Append the child of $u_{t}$ that corresponds to performing action $x^{\star}$ and receiving feedback $m^{\star}$ at time $t$ to the end of $\tilde{P}$, and set $K_{t+1}=\left\{j \in K_{t} \mid m_{j, t}=m^{\star}\right\}$.

Special Update. We apply this rule if $t=d_{i}$ for some $i \in K_{t}$. Let $t^{\prime} \in\left\{d_{i}+1, \ldots, d_{i+1}\right\}$ and $x^{\star} \in\{$ transmit, listen $\}$ be chosen to maximize the probability for a device currently in $u_{t}$ to be idle throughout the time interval $\left[t, t^{\prime}-2\right]$ and to perform $x^{\star}$ at time $t^{\prime}-1$. If $x^{\star}=$ transmit, let $m^{\star}=\lambda_{N}$. Otherwise, let $m^{\star} \in\left\{\lambda_{S}, \lambda_{N}\right\}$ be chosen to maximize the number of indices $j \in K_{t} \backslash\{i\}$ with $m_{j, t^{\prime}-1}=m^{\star}$. We let $u_{t^{\prime}}$ be the unique descendant of $u_{t}$ 

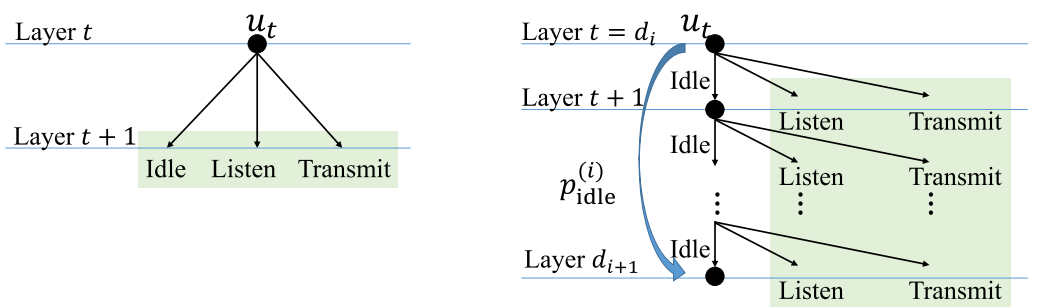

Fig. 4. Left: regular update. Right: special update. The shaded region indicates the set of candidate endpoints to extend the current path $\tilde{P}=\left(u_{1}, \ldots, u_{t}\right)$.

resulting from applying $t^{\prime}-t$ idle actions throughout the time interval $\left[t, t^{\prime}-2\right]$ and then performing action $x^{\star}$ and receiving feedback $m^{\star}$ at time $t^{\prime}-1$. The path $\tilde{P}=\left(u_{1}, \ldots, u_{t}\right)$ is extended to $\tilde{P}=\left(u_{1}, \ldots, u_{t^{\prime}}\right)$. For each $t^{\prime \prime} \in\left\{t+1, \ldots, t^{\prime}-1\right\}$, we set $K_{t^{\prime \prime}}=K_{t} \backslash\{i\}$. For the new endpoint $u_{t^{\prime}}$, we set $K_{t^{\prime}}=\left\{j \in K_{t} \backslash\{i\} \mid m_{j, t^{\prime}-1}=m^{\star}\right\}$.

See Figure 4 for an illustration of the update rules. The reason that $i$ must be removed from the set of the active indices in a special update is that $\mathcal{T}_{i}$ only contains states up to layer $d_{i}$. In what follows, we prove properties of the high energy path $P=\left(u_{1}, \ldots, u_{\hat{t}}\right)$ resulting from the above procedure. For each $t \in\{1, \ldots, \hat{t}\}$, we define the invariant $\mathcal{I}_{t}$ as $u_{t} \in \mathcal{T}_{i}$ for each $i \in K_{t}$. By Lemma 8 , if $\mathcal{I}_{t}$ holds and $\left|K_{t}\right| \geq 2$, then $u_{t}$ is not a terminal state.

Lemma 9. Consider a special update for $\tilde{P}=\left(u_{1}, \ldots, u_{t}\right)$, where $t=d_{i}$. Let s be a device in $u_{t}$. Let $p_{\mathrm{idle}}^{(i)}$ be the probability that s remains idle throughout the time interval $\left[d_{i}, \ldots, d_{i+1}-1\right]$. Suppose that $\mathcal{I}_{t}$ holds. Then $p_{\text {idle }}^{(i)}<1 / 2$.

Proof. Since $\mathcal{I}_{t}$ holds and $i \in K_{t}$, the state $u_{d_{i}}$ belongs to $\mathcal{T}_{i}$. Lemma 7 implies that with probability $1-n_{i}^{-7}$ there is a device $s$ in the state $u_{t}$ when we execute $\mathcal{A}$ on $n_{i}$ devices. With probability $p_{\text {idle }}^{(i)}$, such a device $s$ violates the time constraint $T\left(n_{j}\right)$, since $T\left(n_{j}\right)<d_{j+1}$. Thus, we must have $1 / n_{i} \geq\left(1-n_{i}^{-7}\right) p_{\text {idle }}^{(i)}$, which implies $p_{\text {idle }}^{(i)}<1 / 2$.

LEMma 10. Let $t \in\{1, \ldots, \hat{t}\}$, and let $i \in K_{t}$. If $\mathcal{I}_{\bar{t}}$ holds for all $\bar{t} \in\{1, \ldots, t-1\}$, then $p_{u_{t}}>n_{i}^{-1 / 10}$.

Proof. We first make the following two observations: (i) in a regular update for $\tilde{P}=\left(u_{1}, \ldots, u_{t}\right)$, we have $p_{u_{t+1}} \geq \frac{p_{u_{t}}}{3}$; (ii) in a special update for $\tilde{P}=\left(u_{1}, \ldots, u_{t}\right)$ with $t=d_{j}$, we have $p_{u_{t^{\prime}}} \geq$ $\frac{p_{u_{t}}}{2 \cdot\left(d_{j+1}-d_{j}\right)}\left(1-p_{\text {idle }}^{(j)}\right)>\frac{p_{u_{t}}}{4 d_{j+1}}$. Recall that $u_{t^{\prime}}$ is the new endpoint of $\tilde{P}$ after the special update. Refer to Lemma 9 for the definition of $p_{\text {idle }}^{(i)}<1 / 2$.

Now, fix any $t \in\{1, \ldots, \hat{t}\}$ and $i \in K_{t}$. Notice that $d_{i} \geq t$ and $i=O\left(\log ^{*} d_{i}\right)$. We write $N_{r}<t$ and $N_{s}<i$ to denote the number of regular updates and special updates during the construction of the first $t-1$ states of $P$. In view of the above two observations, we have:

$$
p_{u_{t}} \geq(1 / 3)^{N_{r}} \cdot\left(1 / 4 d_{i}\right)^{N_{s}}>(1 / 3)^{t} \cdot\left(1 / 4 d_{i}\right)^{i}>\left(2^{2^{d_{i}}}\right)^{-1 / 10}>n_{i}^{-1 / 10},
$$

as desired.

Lemma 11. Consider a regular update for $\tilde{P}=\left(u_{1}, \ldots, u_{t}\right)$, and consider any $j \in K_{t}$. Suppose that $u_{t} \in \mathcal{T}_{j}$, and $\mathcal{I}_{\bar{t}}$ holds for all $\bar{t} \in\{1, \ldots, t\}$. If $x^{\star}=$ transmit, then $m^{\star}=\lambda_{N}=m_{j, t}$.

Proof. By Lemma 10, $p_{u_{t+1}}=p_{u_{t}} \cdot p_{u_{t} \rightsquigarrow \text { transmit }}>n_{j}^{-1 / 10}$. Since $u_{t}$, the parent of $u_{t+1}$, is already in $\mathcal{T}_{j}$, according to the definition of $\mathcal{T}_{j}$, we must add $u_{t+1}$ to $\mathcal{T}_{j}$, and set $\lambda_{N}=m_{j, t}$. 
Lemma 12. Consider a special update that extends $\tilde{P}=\left(u_{1}, \ldots, u_{t}\right)$ to $\left(u_{1}, \ldots, u_{t^{\prime}}\right)$, and consider any $j \in K_{t^{\prime}}$. Suppose that $u_{t^{\prime}-1} \in \mathcal{T}_{j}$, and $\mathcal{I}_{\bar{t}}$ holds for all $\bar{t} \in\left\{1, \ldots, t^{\prime}-1\right\}$. If $x^{\star}=$ transmit, then $m^{\star}=\lambda_{N}=m_{j, t^{\prime}-1}$.

Proof. The proof is the same as that of Lemma 11. By Lemma 10, $p_{u_{t^{\prime}}}=p_{u_{t^{\prime}-1}} \cdot p_{u_{t} \rightsquigarrow \text { transmit }}>$ $n_{j}^{-1 / 10}$. Since $u_{t^{\prime}-1}$, the parent of $u_{t^{\prime}}$, is already in $\mathcal{T}_{j}$, according to the definition of $\mathcal{T}_{j}$, we must add $u_{t^{\prime}}$ to $\mathcal{T}_{j}$, and set $\lambda_{N}=m_{j, t^{\prime}-1}$.

Lemma 13. For each $t \in\{1, \ldots, \hat{t}\}, \mathcal{I}_{t}$ holds.

Proof. For the base case, $\mathcal{I}_{1}$ holds trivially. Assume that $\mathcal{I}_{\bar{t}}$ holds for all $\bar{t} \in\{1, \ldots, t-1\}$, we prove that $\mathcal{I}_{t}$ holds. For any $j \in K_{t}$, we show that $u_{t} \in \mathcal{T}_{j}$.

Suppose that $u_{t}$ is resulting from applying action $x$ and hearing the channel feedback $m$. By Lemma 10, $p_{u_{t}}=p_{u_{t-1}} \cdot p_{u_{t} \rightsquigarrow x}>n_{j}^{-1 / 10}$. Since $K_{t} \subseteq K_{t-1}$, by induction hypothesis, $u_{t-1} \in \mathcal{T}_{j}$. In what follows, we do a case analysis for all choices of $x \in$ \{transmit, listen, idle\}.

If $x=$ idle, then $u_{t}$ must be in $\mathcal{T}_{j}$, regardless of the choice of $m_{j, t-1}$, according to the definition of $\mathcal{T}_{j}$. If $x=$ listen, then according to the construction of $P$, we have $m=m_{j, t-1}$, and so $u_{t}$ is in $\mathcal{T}_{j}$. If $x=$ transmit, we have $m=\lambda_{N}$ by the construction of $P$, and $m=\lambda_{N}=m_{j, t-1}$ due to Lemma 11 and Lemma 12, and so $u_{t}$ is in $\mathcal{T}_{j}$.

We are now in a position to prove the main result of this section.

LEMMA 14. For any positive integer $k$, there is a network size $n$ satisfying $d_{1} \leq n \leq d_{k+1}$ such that in an execution of $\mathcal{A}$ on $n$ devices, with probability at least $1-n^{-7}$, there is a device that performs $\Omega(\log k)$ listen steps.

Proof. First, we bound the shrinkage rate of the size of active indices $K_{t}$. Consider a regular update for $\tilde{P}=\left(u_{1}, \ldots, u_{t}\right)$. If $x^{\star}=$ idle, then $K_{t+1}=K_{t}$. If $x^{\star}=$ transmit, then we also have $K_{t+1}=\left\{j \in K_{t} \mid m_{j, t}=m^{\star}\right\}=K_{t}$ in view of Lemma 11. If $x^{\star}=$ listen, then our choice of $m^{\star}$ in the regular update implies $\left|K_{t+1}\right| \geq\left|K_{t}\right| / 2$. Next, consider a special update that extends $\tilde{P}=\left(u_{1}, \ldots, u_{t}\right)$ to $\left(u_{1}, \ldots, u_{t^{\prime}}\right)$. Similarly, if $x^{\star} \in\{$ idle, transmit $\}$, then $K_{t^{\prime}}=K_{t} \backslash\{i\}$, where $i$ is the index such that $t=d_{i}$; see Lemma 12. For the case of $x^{\star}=$ listen, our choice of $m^{\star}$ in the special update implies $\left|K_{t^{\prime}}\right| \geq\left(\left|K_{t}\right|-1\right) / 2$. Therefore, any device whose execution history following the path $P=\left(u_{1}, \ldots, u_{\hat{t}}\right)$ performs $\Omega\left(\log \left|K_{1}\right|-\log \left|K_{\hat{t}}\right|\right)$ listen steps.

The stopping criterion, together with our calculation of the shrinkage rate of $\left|K_{t}\right|$, implies that $\left|K_{\hat{t}}\right| \geq 2$. We let $\hat{i}$ be any element in $K_{\hat{t}}$, and set $n=n_{\hat{i}}$. By Lemma $13, u_{\hat{t}} \in \mathcal{T}_{\hat{i}}$. Then, Lemma 7 implies that in an execution of $\mathcal{A}$ on $n_{\hat{i}}$ devices, with probability $1-n_{\hat{i}}^{-7}$, at least $n_{\hat{i}} \cdot p_{u_{\hat{t}}}-(\hat{t}-$ 1) $\cdot n_{\hat{i}}^{0.6}=\Omega\left(n_{\hat{i}}^{0.9}\right)>1$ devices enter the state $u_{\hat{t}}$ along the path $P$, and any such device performs $\Omega\left(\log \left|K_{1}\right|-\log \left|K_{\hat{t}}\right|\right)=\Omega(\log k)$ listen steps.

Similarly, so long as $T(n) \leq \exp ^{(\ell)}(n)$, for some constant $\ell$, where $\exp ^{(i)}$ is iterated $i$-fold application of exp, it is possible to set the checkpoints such that $k=\Theta\left(\log ^{*}\left(d_{k+1}\right)\right)$, and so Lemma 14 implies that the energy cost $\mathcal{A}$ is $\Omega\left(\log \log ^{*} n\right)$. Therefore, we conclude Theorem 4 .

\subsection{Other Problems}

In this section, we discuss lower bounds of other problems.

Successful Communication. We demonstrate how our lower bounds proofs can be adapted to the class $C$ of all problems that require each device to perform at least one successful communication before it terminates. In particular, this includes Leader Election and the contention resolution problem studied in Reference [7]. Notice that Approximate Counting, in general, does not require each device to perform a successful communication before it terminates. 
Consider the Sender-CD model. Let $\mathcal{A}$ be a polynomial time algorithm, and a device in an execution of $\mathcal{A}$ must perform at least one successful communication before it terminates. Let the runtime of $\mathcal{A}$ be $T(n)$. Let $n=n_{i}$ for some $i$. Consider a device $s$ in an execution of $\mathcal{A}$ on $n_{i}$ devices. Let $t_{\text {suc }}$ be time of the first successful communication of $s$. Then $t_{\text {suc }} \leq T\left(n_{i}\right)<d_{i+1}$ with probability $1-1 / n_{i}$. By Lemma 3 , with probability $1-n_{i}^{-7}$, by time $d_{i}$ all devices are confined in $\mathcal{T}_{i} \subseteq \mathcal{T}_{\text {no-comm }}$ and no successful communication occurs throughout the time interval $\left[1, d_{i}-1\right]$. Thus, $t_{\text {suc }} \geq d_{i}$ with probability $1-n_{i}^{-7}$. Since $t_{\text {suc }}$ is within $\left[d_{i}, d_{i+1}\right)$ with probability $1-1 / \operatorname{poly}\left(n_{i}\right)$, this number can be seen as a very loose estimate of the network size $n=n_{i}$, but this estimate is already good enough for the device $s$ to distinguish $n=n_{i}$ from other candidate network sizes in $\left\{n_{j}\right\}$. Since we only consider the set of network sizes $\left\{n_{j}\right\}$ in our proof for Theorem 3 , the proof applies to $\mathcal{A}$. For the same reason, Theorem 4 also applies to all problems in the class $C$ in Strong-CD.

Loneliness Detection. We consider the loneliness detection problem whose goal is to distinguish between $n=1$ and $n>1$; see References [22,23]. We show that this problem is impossible to solve in No-CD. Intuitively, in No-CD, a transmitter cannot simultaneously listen to the channel, and so a device never receives any feedback from the channel if $n=1$. However, when $n$ is large enough relative to $t$, with high probability a device also does not hear any message in the first $t$ time slots. It seems hopeless to have an algorithm that detects loneliness.

Let $T(n)$ be any time function. Let $\mathcal{A}$ be any algorithm in No-CD that accomplishes the following: If $n>1$, with probability at least $1-1 / n$, all devices terminate by time $T(n)$ and output " $n>1$." If $n=1$, then the only participating device $s$ terminates by time $t$ and outputs " $n=1$ " with probability $p$. We show that either $t=\infty$ or $p=0$.

We simulate $\mathcal{A}$ in Sender-CD and apply the analysis in Section 3.2. Recall that in No-CD a transmitter cannot simultaneously listen to the channel, and so for each terminal state $u \in \mathcal{T}$ $\mathcal{T}_{\text {no-comm }}$ such that the path $P$ leading to $u$ does not involve successfully listening to a message, the output of $u$ is identical to some state $u^{\prime} \in \mathcal{T}_{\text {no-comm }}$ (which results from changing each successful transmission to a failed transmission in the execution history).

For each state $u \in \mathcal{T}_{\text {no-comm }}$, there exists an index $i$ such that $u \in \mathcal{T}_{i}$. By Lemma 3 , in an execution of $\mathcal{A}$ on $n_{i}$ devices, with probability $1-n_{i}^{-7}$, there is at least one device entering the state $u$. Thus, no state in $\mathcal{T}_{\text {no-comm }}$ is a terminal state with output " $n=1$." However, in No-CD with $n=1$ there is no means for a listener to receive a message, and so we must have either $t=\infty$ or $p=0$.

\section{DETERMINISTIC UPPER BOUND}

In this section, we present an optimal deterministic algorithm for Census in Sender-CD that simultaneously matches the $\Omega(\log \log N)$ energy lower bound of Theorem 2 and the $\Omega(N)$ time lower bound of Reference [27, Theorem 1.6]. Notice that any Census algorithm also solves Leader Election.

Theorem 5. There exists a deterministic Sender-CD algorithm that solves Census in $O(N)$ time with energy $O(\log \log N)$.

Our algorithm is inspired by an energy-sharing technique introduced in Reference [27], which is based on the concept of groups. We call an ID active if there is a device of such an ID; we also write $s$ to denote the device of ID $s$.

A group $G$ is a set of active IDs meeting the following criteria: Each device belongs to at most one group. Let $G=\left(s_{1}, \ldots, s_{k}\right)$ be the members of $G$, listed in increasing order by ID. The rank of a device $s_{i} \in G$ is defined as $i$. We assume each group $G$ has a unique group ID. Similarly, we say that a group ID $x$ is active if there is a group $G$ whose ID is $x$. Each group $G$ has a device $s \in G$ that serves as the representative of $G$. We allow the representative of a group to be changed over time. 
Each device $s \in G$ knows (i) the group ID of $G$, (ii) the current representative of $G$, and (iii) the list of all IDs in $G$.

\subsection{A Simple Census Algorithm}

In this section, we show how to use groups to distribute energy costs to devices. Consider the following setting: All devices are partitioned into groups whose IDs are within the range $\{1, \ldots, \hat{N}\}$, and each group has size at least $g$. We present a No-CD deterministic algorithm $\operatorname{SimpleCensus}(\hat{N}, g)$ that elects a leader group $G^{\star}$ such that the representative of $G^{\star}$ knows the list of IDs of all devices. The algorithm $\operatorname{SimpleCensus}(\hat{N}, g)$ ends when the representative of $G^{\star}$ announces the list of IDs of all devices; the last time slot of SimpleCensus $(\hat{N}, g)$ is called the "announcement time slot." The algorithm SimpleCensus $(\hat{N}, g)$ is executed recursively, as follows:

Base Case. If $\hat{N}=1$, then there is only one group $G$ that contains all devices. By definition, each device in $G$ already knows the list of IDs of all devices in $G$. We set $G^{\star}=G$ and let the representative of $G$ announce the list of IDs of all devices in $G$ at the announcement time slot.

Inductive Step. Assume $\hat{N}>1$. The algorithm invokes two recursive calls. For each group $G$, if the rank of the current representative of $G$ is $i$, then the representative of $G$ in a recursive call will be the device of rank $i+1$ (or 1 if $i=|G|$ ). The two recursive calls are made on the two halves of the ID space, $S_{1}=\{1, \ldots,\lceil\hat{N} / 2\rceil\}$ and $S_{2}=\{\lceil\hat{N} / 2\rceil+1, \ldots, \hat{N}\}$. The representative $s$ of each group $G$ listens to the announcement time slots of the two recursive calls; after that, $s$ learns the list of IDs of all devices. The leader group $G^{\star}$ is selected as the one that contains the device of the smallest ID. Then, we let the representative of $G^{\star}$ announce the list of IDs of all devices at the announcement time slot.

It is straightforward to see that the algorithm $\operatorname{SimpleCensus}(\hat{N}, g)$ takes $O(\hat{N})$ time and uses $O\left(\left\lceil\frac{\log \hat{N}}{g}\right\rceil\right)$ energy. We summarize the result as a lemma.

LEMMA 15. Suppose that all devices are partitioned into groups whose IDs are within the range $\{1, \ldots, \hat{N}\}$, and each group has size at least $g$. The algorithm SimpleCensus $(\hat{N}, g)$ elects a leader group $G^{\star}$ such that the representative of $G^{\star}$ knows the list of IDs of all devices. The algorithm takes $O(\hat{N})$ time and uses $O\left(\left\lceil\frac{\log \hat{N}}{g}\right\rceil\right)$ energy.

\subsection{An Optimal Census Algorithm}

In this section, we prove Theorem 5. Without loss of generality, we assume that $\log N$ is an integer. Our algorithm consists of $O(\log \log N)$ phases. All devices participate initially and may drop out in the middle of the algorithm. We maintain the following invariant $\mathcal{I}_{i}$ at the beginning of the $i$ th phase:

Invariant $\mathcal{I}_{i}$. (i) All participating devices are partitioned into groups of size exactly $2^{i-1}$ in the group ID space $\{1, \ldots, N\}$. (ii) For each device that drops out during the first $i-1$ phases, its ID is remembered by the representative of at least one group.

Termination. There are two different outcomes of our algorithm. For each index $i$, the algorithm terminates at the beginning of the $i$ th phase if either (i) there is only one group remaining, or (ii) $i=\log \log N+1$.

Suppose that at the beginning of the $i$ th phase there is only one group $G$ remaining; then the algorithm is terminated with the representative of $G$ knowing the list of IDs of all devices. Suppose that more than one group remains at the beginning of the $(\log \log N+1)$ th phase; as the groups that survive until this moment have size $\log N$, we can apply $\operatorname{SimpleCensus}(N, \log N)$ to solve Census in $O(N)$ time with $O(1)$ energy cost. 
Overview. At the beginning of the first phase, each device $s$ forms a singleton group $G=\{s\}$, and so the invariant $\mathcal{I}_{1}$ is trivially met. Throughout the algorithm, the group ID of a group $G$ is always defined as the minimum ID of the devices in $G$.

During the $i$ th phase, each group attempts to find another group to merge into a group with size $2^{i}$. Each group $G$ that is not merged drops out, and the list of IDs in $G$ is remembered by the representative of at least one other group $G^{\prime}$ that does not drop out. In what follows, we describe the algorithm for the $i$ th phase. At the beginning of the $i$ th phase, it is guaranteed that the invariant $\mathcal{I}_{i}$ is met. We also assume that the number of groups is at least 2, since otherwise the terminating condition is met.

Step 1-Merging Groups. The first step of the ith phase consists of the procedure $\operatorname{Det} L E(N)$, which costs $O(N)$ time and $O(\log \log N)$ energy. For each device $s$, we write $I(s)$ to denote the set of all IDs that the device $s$ has heard during the first $i-1$ phases (including the ID of $s$ ). Notice that $I(s) \supseteq G$ if $s$ belongs to the group $G$. The procedure $\operatorname{Det} L E(\hat{N})$ is defined recursively as follows:

Base Case. Suppose that the group ID space $S$ has size $\hat{N}=2$, and there are exactly two groups $G_{1}$ and $G_{2}$. Using two time slots, the representatives $s_{1}$ and $s_{2}$ of the two groups exchange the information $I\left(s_{1}\right)$ and $I\left(s_{2}\right)$, and then the two groups are merged.

Inductive Step. Suppose that the group ID space $S$ has size $\hat{N}>2$, and there are at least two groups. Uniformly divide the group ID space $S=\{1, \ldots, \hat{N}\}$ into $N^{\prime}=\lceil\sqrt{\hat{N}}\rceil$ intervals $S_{1}, \ldots, S_{N^{\prime}}$, and each of them has size at most $N^{\prime}=\lceil\sqrt{\hat{N}}\rceil$.

For each $j \in\left\{1, \ldots, N^{\prime}\right\}$, let $z_{j}$ be the number of groups whose ID is within $S_{j}$. In the Sender-CD model, testing whether $z_{j}=1$ can be done by letting the representatives of all groups whose ID are in $S_{j}$ speak simultaneously. If $z_{j} \neq 1$, invoke a recursive call to $\operatorname{Det} \operatorname{LE}\left(N^{\prime}\right)$ on the group ID space $S_{j}$; the recursive call is vacuous if $z=0$.

Let $\mathcal{G}$ be the set of all groups that do not participate in the above recursive calls. That is, $G \in \mathcal{G}$ if the ID of $G$ belongs to an interval $S_{j}$ with $z_{j}=1$. In the Sender-CD model, we can check whether $|\mathcal{G}|=1$ in one time slot by letting the representatives of groups in $\mathcal{G}$ speak simultaneously. For the case of $|\mathcal{G}| \neq 1$, we invoke a recursive call to $\operatorname{DetLE}\left(N^{\prime}\right)$ on $\mathcal{G}$, where the ID space is $S^{\prime}=$ $\left\{1, \ldots, N^{\prime}\right\}$ and the group ID of the group from $S_{j}$ is $j$. For the case of $|\mathcal{G}|=1$, we allocate one time slot to let the representative $s$ of the unique group $G \in \mathcal{G}$ announce $I(s)$ to the representatives of all other groups, and then $G$ drops out from the algorithm.

Analysis. By the end of $\operatorname{Det} \operatorname{LE}(\hat{N})$, for each group $G$ whose representative is $s$, we have (i) $G$ is merged with some other group $G^{\prime}$, or (ii) $G$ drops out, and $I(s)$ is remembered by the representative $s^{\prime}$ of some other group $G^{\prime}$.

Let $E(\hat{N})$ and $T(\hat{N})$ denote the energy complexity and the time complexity of $\operatorname{Det} \operatorname{E}(\hat{N})$ on group ID space of size $\hat{N}$. We have $T(2)=E(2)=O(1)$ and

$$
\begin{aligned}
& E(\hat{N})=E(\lceil\sqrt{\hat{N}}\rceil)+O(1) \\
& T(\hat{N})=(\lceil\sqrt{\hat{N}}\rceil+1) \cdot T(\lceil\sqrt{\hat{N}}\rceil)+O(\lceil\sqrt{\hat{N}}\rceil) .
\end{aligned}
$$

It is straightforward to show that $E(\hat{N})=O(\log \log \hat{N})$ and $T(\hat{N})=O(\hat{N})$.

Step 2-Disseminating Information and Electing New Representatives. Notice that only the representatives of the groups participate in Step 1. Let $G$ be a group whose representative is $s$. If $G$ is merged with some other group $G^{\prime}$ whose representative is $s^{\prime}$, then we need all members in $G$ to know $I\left(s^{\prime}\right)$ and the list of members in $G^{\prime}$. If $G$ decides to drop out from the algorithm, then we need all members in $G$ to know about this information. We allocate $N$ time slots for the representatives 
to communicate with other group members. The energy cost for the information dissemination is $O(1)$ per device.

To save energy, we need each device to serve as a representative for not too many phases. For the first phase, each device $s$ inevitably serves as the representative of its group $G=\{s\}$. For $i>1$, for each group $G$ participating in the $i$ th phase, there must be some member of $G$ that has only served as a representative once. Among all members of $G^{\prime}$ that have only served as a representative once, we let the one that has the minimum ID to be the representative of $G$ during the $i$ th phase. Therefore, each device serves as a representative for at most two phases throughout the entire algorithm.

Time and Energy Complexity. We analyze the runtime and the energy cost of the entire algorithm. The energy cost for a device $s$ in one phase is $O(\log \log N)$ if $s$ serves as a representative, and is $O(1)$ otherwise. There are $O(\log \log N)$ phases, and each device serves as a representative for no more than two phases throughout the algorithm. Therefore, the energy cost of the algorithm is $O(\log \log N)$ per device. The runtime of the algorithm is $O(N \log \log N)$, since each phase takes $O(N)$ time. The runtime can be further reduced to $O(N)$ by doing the following preprocessing step: Uniformly divide the ID space into $\frac{N}{\log \log N}$ intervals, and call SimpleCensus $(\log \log N, 1)$ on each interval. This takes $O(N)$ time and $O(\log \log \log N)=o(\log \log N)$ energy. After the preprocessing, each interval has a leader that knows the list of IDs of all devices in the interval. We only let the leaders of the $\frac{N}{\log \log N}$ intervals participate in our algorithm. This reduces the size of the ID space from $N$ to $N^{\prime}=\frac{N}{\log \log N}$, and so the runtime is improved to $O\left(N^{\prime} \log \log N^{\prime}\right)=O(N)$.

\section{DETERMINISTIC UPPER BOUND FOR DENSE INSTANCES}

In this section, we present a deterministic algorithm that solves Census with inverse Ackermann energy cost when the input is dense in the ID space, i.e., the number of devices $n$ is at least $c \cdot N$ for a fixed constant $c>0$. This improves upon a prior work of Jurdzinski et al. [27] that uses $O\left(\log ^{*} N\right)$ energy. For any two positive integers $i$ and $j$, we define the two functions $a_{i}(j)$ and $b_{i}(j)$ as follows:

$$
a_{i}(j)=\left\{\begin{array}{ll}
j^{9} & \text { if } i=1, \\
a_{i-1}^{(j)}\left(j^{8}\right) & \text { if } i>1,
\end{array} \quad b_{i}(j)= \begin{cases}2^{j} & \text { if } i=1, \\
2^{j} \prod_{r=0}^{j-1} b_{i-1}\left(a_{i-1}^{(r)}\left(j^{8}\right)\right) & \text { if } i>1 .\end{cases}\right.
$$

The notation $f^{(r)}$ is iterated $r$-folded application of $f$, which is defined as $f^{(0)}(x)=x$ and $f^{(r)}(x)=$ $f\left(f^{(r-1)}(x)\right)$. We define the inverse Ackermann function $\alpha(N)$ to be the minimum number $i$ such that $b_{i}(55) \geq N$. This is not the standard definition of $\alpha$, but it is identical to any other definition from the literature, up to $\pm O(1)$. The goal of this section is to prove the following theorem:

Theorem 6. Suppose the number of devices $n$ is at least $c \cdot N$ for a fixed constant $c>0$. There is a deterministic No-CD algorithm that solves Census in time $O(N)$ with energy cost $O(\alpha(N))$.

Our algorithm is based on the recursive subroutine DenseAlgo ${ }_{i}(\hat{N}, j)$, which is capable of merging groups into fewer and larger ones using very little energy. The parameter $i$ is a positive integer indicating the height of the recursion. The parameter $\hat{N}$ is an upper bound on the size of the group ID space; for technical reasons, we allow $\hat{N} \geq 1$ to be a fractional number. The parameter $j$ is a lower bound on the group size; we assume $j \geq 55$. The precise specification of DenseAlgo ${ }_{i}(\hat{N}, j)$ is as follows:

Input. Prior to the execution of DenseAlgo $(\hat{N}, j)$, the set of all devices are partitioned into groups. The size of the group ID space is at most $\hat{N}$. Each group has size at least $j$. The total number of groups is at least $\hat{N} / \log j$.

Output. Some devices drop out during the execution of $\operatorname{DenseAlgo}_{i}(\hat{N}, j)$. The fraction of the devices that drop out is at most $2 / j$ of all devices. After the execution of DenseAlgo ${ }_{i}(\hat{N}, j)$, 
the remaining devices form new groups. The size of the group ID space is at most $N^{\prime}=$ $\max \left\{1, \hat{N} / b_{i}(j)\right\}$. Each group has size at least $a_{i}(j)$. The total number of groups is at least $N^{\prime} / \log a_{i}(j)$. We allocate $N^{\prime}$ "announcement time slots" at the end of DenseAlgo $(\hat{N}, j)$. At the $k$ th announcement time slot, the representative of the group $G$ of ID $k$ announces the list of all members of $G$.

We have more stringent requirements for the case of $i=1$ : (i) the fraction of the devices that drop out is at most $1 / j$ of all devices, and (ii) the total number of groups is at least $N^{\prime} / 8 \log j$.

Complexity. The procedure DenseAlgo ${ }_{i}(\hat{N}, j)$ takes $O(\hat{N})$ time and consumes $O(i)$ energy per device.

In Sections 5.1 and 5.2, we present and analyze the subroutine DenseAlgo ${ }_{i}(\hat{N}, j)$. We have the following auxiliary lemma:

Lemma 16. Suppose the ID space of devices is $S=\{1, \ldots, N\}$, and there is a group $G$ of size $\epsilon N$. Then there is a deterministic algorithm that solves Census in $O(N)$ time and $O(1 / \epsilon)$ energy.

Proof. The algorithm is as follows: Partition the ID space $S$ into $k=\epsilon N=|G|$ intervals $S_{1}, \ldots, S_{k}$, where each interval has size at most $\lceil 1 / \epsilon\rceil$. Let $s_{i}$ be the device in $G$ that is of rank $i$, and let $L_{i}$ be the list of IDs of all devices in $S_{i}$. For each $1 \leq i \leq k$, we let $s_{i}$ learn $L_{i}$ by having $s_{i}$ listens for $\left|S_{i}\right|=O(1 / \epsilon)$ time slots, where each device in $S_{i}$ transmits once. Next, we allocate $k-1$ time slots to do the following: For $i=1$ to $k-1$, let $s_{i}$ transmit $\bigcup_{j=1}^{i} L\left(s_{j}\right)$ and $s_{i+1}$ listen. After that, $s_{k}$ knows the list of IDs of all devices, and we let $s_{k}$ announce the list while all other devices listen to the channel.

We are now in a position to prove Theorem 6 . The proof is based on Lemma 16 and the procedure $\operatorname{DenseAlgo}_{i}(\hat{N}, j)$. Recall that the number of devices is promised to be at least $c N$. We choose $j^{*}=\max \left\{55,2^{\lceil 1 / c\rceil}\right\}$ and let $i^{*}$ be the minimum number $i$ such that $N / b_{i}\left(j^{*}\right) \leq 1$. To artificially satisfy the input invariant, we imagine that each device simulates a group of $\log j^{*}=O(1)$ devices. Notice that the group ID space is $\{1, \ldots, N\}$, and the total number of groups is $n \geq c N \geq N / \log j^{*}$. Thus, the requirement for executing DenseAlgo $i_{i^{*}}\left(N, j^{*}\right)$ is met. We execute DenseAlgo $i_{i^{*}}\left(N, j^{*}\right)$, which costs $O(N)$ time and $O\left(i^{*}\right)=O(\alpha(N))$ energy. During the execution, at most $2 / j^{*}$ fraction of devices drop out. All remaining devices form $1=\max \left\{1, N / b_{i^{*}}\left(j^{*}\right)\right\}$ group. After that, we can solve Census by the algorithm of Lemma 16 using additional $O(N)$ time and $O(1)$ energy.

\subsection{Base Case}

In this section, we present the base case $i=1$ of the subroutine $\operatorname{DenseAlgo}_{i}(\hat{N}, j)$ and show that it meets the required specification. At the beginning, all devices are organized into groups of size at least $j$, and the group ID space $S$ has size at most $\hat{N}$. We partition the group ID space $S$ into $k=\left\lceil\hat{N} / 2^{j+1}\right\rceil$ intervals $S_{1}, \ldots, S_{k}$ such that each interval has size at most $2^{j+1}$. For each interval $S_{l}$, we run SimpleCensus $\left(\left|S_{l}\right|, j\right)$ to merge all groups in the interval $S_{l}$ into a $\operatorname{single}$ group $G$, and let $l$ be the ID of $G$. After that, for each group $G$ of size less than $j^{9}$, all devices in $G$ drop out. The execution of SimpleCensus $\left(\left|S_{l}\right|, j\right)$ takes $O\left(\left|S_{l}\right|\right)$ time and $O(1)$ energy. Thus, algorithm DenseAlgo ${ }_{1}(\hat{N}, j)$ costs $O(\hat{N})$ time and $O(1)$ energy. It is clear that each group in the output has size at least $j^{9}=a_{1}(j)$. We show that the output meets the remaining requirements.

Size of Group ID Space. The size of the output group ID space is $k=\left\lceil\hat{N} / 2^{j+1}\right\rceil$. For the case of $\hat{N} / 2^{j+1} \leq 1$, we have $k=1$. For the case of $\hat{N} / 2^{j+1}>1$, we have $k=\left\lceil\hat{N} / 2^{j+1}\right\rceil<$ $\hat{N} / 2^{j}=\hat{N} / b_{1}(j)$. Thus, the size of the group ID space $k$ is always upper bounded by $N^{\prime}=\max \left\{1, \hat{N} / b_{1}(j)\right\}$. 
Proportion of Terminated Devices. The number of devices that are terminated is at most $z_{\text {term }}=\left(j^{9}-1\right) k<j^{9} \hat{N} / 2^{j}$. The total number of devices is at least $z_{\text {init }}=j \hat{N} / \log j$. Thus, the proportion of the terminated devices is at most $f=z_{\text {term }} / z_{\text {init }}=\frac{j^{8} \log j}{2^{j}}$. As long as $j \geq 55$, we have $f<1 / j$.

Number of Groups. The total number of devices is at least $z_{\text {init }}=j \hat{N} / \log j$. The size of each output group is at most $j 2^{j+1}$. Since the proportion of the terminated devices is at most $1 / j \leq 1 / 55<1 / 2$, the number of output groups is at least

$$
z_{\text {out }}=\max \left\{1,\left\lfloor\left(z_{\text {init }} / 2\right) /\left(j 2^{j+1}\right)\right\rfloor\right\}=\max \left\{1,\left\lfloor z_{\text {init }} /\left(j 2^{j+2}\right)\right\rfloor\right\} .
$$

We show that the inequality $z_{\text {out }} \geq \max \left\{1, \hat{N} /\left(2^{j} 8 \log j\right)\right\} \geq N^{\prime} / 8 \log j$ holds. For the case of $z_{\text {out }}=1$, the inequality is already met. If $z_{\text {out }}>1$, we have $z_{\text {out }}=\left\lfloor z_{\text {init }} /\left(j 2^{j+2}\right)\right\rfloor \geq$ $z_{\text {init }} /\left(j 2^{j+3}\right)=\hat{N} /\left(2^{j} 8 \log j\right)$, as desired.

\subsection{Inductive Step}

In this section, we consider the case of $i>1$. The algorithm DenseAlgo ${ }_{i}(\hat{N}, j)$ begins with an initialization step, which increases the group size from $j$ to $j^{9}$ by executing DenseAlgo ${ }_{1}(\hat{N}, j)$. After that, it recursively invokes DenseAlgo ${ }_{i-1}\left(X_{r}, Y_{r}\right)$, for $r$ from 1 to $j^{\star}$, where $j^{\star}$ and the sequences $\left(X_{r}\right)_{r \in\left[j^{\star}\right]}$ and $\left(Y_{r}\right)_{r \in\left[j^{\star}\right]}$ will be determined. Each device participates in the initialization step and exactly one recursive call to DenseAlgo ${ }_{i-1}$, so the energy cost per device is $O(1)+O(i-1)=O(i)$.

After the initialization step, each group $G$ has size $j^{9}$. For each group $G$, we extract $j^{\star}$ subgroups $G_{1}, G_{2}, \ldots, G_{j^{\star}}$ from the members of $G$, each with size exactly $j^{8}$ (we will later see that $j^{\star} \leq j$ ). The subgroup $G_{r}$ is responsible for representing $G$ in the $r$ th recursive call DenseAlgo ${ }_{i-1}\left(X_{r}, Y_{r}\right)$. For $1 \leq r<j^{\star}$, as $G_{r}$ and $G_{r+1}$ have the same size, we set up a bijection $\phi_{r}: G_{r} \rightarrow G_{r+1}$. For each device $s$ in the $r$ th subgroup $G_{r}$, after $s$ finishes the $r$ th recursive call, if $s$ has not dropped out yet, $\phi_{r}(s)$ continues to play the role of $s$ in the $(r+1)$ th recursive call. $\phi_{r}(s)$ learns all information known to $s$ by listening to an announcement time slot of the $r$ th recursive call.

Parameters of Recursive Calls. If $\hat{N} / 2^{j}<2$, then only one group remains after the initialization step DenseAlgo ${ }_{1}(\hat{N}, j)$, and so we are already done without doing any more recursive calls. In what follows, we assume $\hat{N} / 2^{j} \geq 2$. The two sequences $\left(X_{r}\right)_{r \in\left[j^{\star}\right]}$ and $\left(Y_{r}\right)_{r \in\left[j^{\star}\right]}$ are defined as follows: We choose $j^{\star}$ as $\min \left\{j \text {, arg } \min _{r}\left(X_{r+1}<2\right)\right\}^{3}$

$$
X_{r}=\left\{\begin{array}{ll}
\hat{N} / 2^{j} & \text { if } r=1, \\
X_{r-1} / b_{i-1}\left(Y_{r-1}\right) & \text { if } r>1,
\end{array} \quad Y_{r}= \begin{cases}j^{8} & \text { if } r=1, \\
a_{i-1}\left(Y_{r-1}\right) & \text { if } r>1 .\end{cases}\right.
$$

We verify that the requirement of executing the $r$ th recursive call is met, for each $1 \leq r \leq j^{\star}$.

Base Case. For $r=1$, we show that the requirement of DenseAlgo ${ }_{i-1}\left(X_{1}, Y_{1}\right)$ is met after the initialization step DenseAlgo ${ }_{1}(\hat{N}, j)$ : (i) the number of groups is at least $\frac{\hat{N} / 2^{j}}{8 \log j}=X_{1} / \log Y_{1}$; (ii) the size of each group is $j^{8}=Y_{1}$; (iii) the group ID space is at most $\hat{N} / 2^{j}=X_{1}$.

Inductive Step. For $1<r \leq j^{\star}$, we show that the requirement of DenseAlgo ${ }_{i-1}\left(X_{r}, Y_{r}\right)$ is met after the previous recursive call DenseAlgo ${ }_{i-1}\left(X_{r-1}, Y_{r-1}\right)$ : (i) the number of groups is at least $\frac{X_{r-1} / b_{i-1}\left(Y_{r-1}\right)}{\log a_{i-1}\left(Y_{r-1}\right)}=X_{r} / \log Y_{r}$; (ii) the size of each group is $a_{i-1}\left(Y_{r-1}\right)=Y_{r}$; (iii) the group ID space is at most $X_{r-1} / b_{i-1}\left(Y_{r-1}\right)=X_{r}$.

It is also straightforward to see that the output (group size, number of groups, and group ID space size) of the last recursive call DenseAlgo ${ }_{i-1}\left(X_{j^{\star}}, Y_{j^{\star}}\right)$ already satisfies the requirement of

\footnotetext{
${ }^{3}$ We will later see that $X_{r}$ represents the input group ID space for executing the $r$ th recursive call. If $X_{r+1}<2$ for some $r<j$, then we can terminate after the $r$ th recursive call.
} 
the output of DenseAlgo ${ }_{i}(\hat{N}, j)$, since we have $X_{j} / b_{i-1}\left(Y_{j}\right)=\hat{N} / b_{i}(j)$ and $a_{i-1}\left(Y_{j}\right)=a_{i}(j)$. Next, we show that the number of devices that drop out during the execution of DenseAlgo $\sin _{i}(\hat{N}, j)$ is at most $2 / j$ of all devices. Let $f_{i}(j)$ be the fraction of devices the are terminated during the execution of DenseAlgo ${ }_{i}(\hat{N}, j)$. The analysis in Section 5.1 implies that $f_{1}(j) \leq \frac{1}{j}$. We prove that $f_{i}(j) \leq \frac{2}{j}$.

$$
\begin{array}{rlr}
1-f_{i}(j) & \geq\left(1-f_{1}(j)\right) \prod_{r=1}^{j^{\star}}\left(1-f_{i-1}\left(Y_{r}\right)\right) \\
& \geq(1-1 / j) \prod_{r=1}^{j^{\star}}\left(1-2 / Y_{r}\right) & \text { (by induction hypothesis) } \\
& \geq(1-2 / j) .
\end{array}
$$

Energy Complexity. During DenseAlgo ${ }_{i}(\hat{N}, j)$, each device uses $O(1)$ energy in the initialization step. Consider a device $s$ participating in the $r$ th recursive call. If $r>1$, then $s$ uses $O(1)$ energy to learn the information of $\phi_{r-1}^{-1}(s)$. The execution of DenseAlgo ${ }_{i-1}\left(X_{r}, Y_{r}\right)$ costs $O(i-1)$ energy. Thus, each device spends $O(i)$ energy during the execution of DenseAlgo ${ }_{i}(\hat{N}, j)$.

Time Complexity. Let $T_{i}(\hat{N})$ be the runtime of $\operatorname{DenseAlgo}_{i}(\hat{N}, j)$ (for any $j$ ). The analysis in Section 5.1 implies that $T_{1}(\hat{N}) \leq C \hat{N}$ for some constant $C$. We prove that $T_{i}(\hat{N}) \leq 10 C \hat{N}=O(\hat{N})$, for all $i$.

$$
\begin{aligned}
T_{i}(\hat{N}) & =T_{1}(\hat{N})+\sum_{r=1}^{j^{\star}} T_{i-1}\left(X_{r}\right) \\
& \leq C \hat{N}+\sum_{r=1}^{j^{\star}}(10 C) X_{r} \quad \text { (by induction hypothesis) } \\
& =C \hat{N}+10 C \sum_{r=1}^{j^{\star}} X_{r} \\
& \leq C \hat{N}+10 C \cdot 0.9 \hat{N} \\
& =10 C \hat{N} .
\end{aligned}
$$

To summarize, DenseAlgo ${ }_{i}(\hat{N}, j)$ costs $O(\hat{N})$ time and $O(i)$ energy, and the constant hidden in $O(\hat{N})$ is an absolute constant independent of $i$.

\section{RANDOMIZED UPPER BOUNDS}

In this section, we present randomized algorithms for Approximate Counting matching the energy complexity lower bound proved in Section 3. In Reference [7], a randomized algorithm for Approximate Counting in Strong-CD using $O\left(\log \left(\log ^{*} n\right)\right)$ energy is devised. They showed that any circuit of constant fan-in, with input bits encoded as noise $=1$ and silence $=0$, can be simulated with $O(1)$ energy cost, and an estimate of the network size can be computed by such a circuit. The circuit simulation of Reference [7] makes extensive use of collision detection. In this section, we demonstrate a different approach to Approximate Counting based on our dense Census algorithm, which can be implemented in all four collision detection models.

THEOREM 7. There is an algorithm that, with probability $1-1 / \operatorname{poly}(n)$, solves Approximate Counting in $n^{o(1)}$ time with energy cost $O\left(\log ^{*} n\right)$ if the model is Sender-CD or No-CD, or $O\left(\log \left(\log ^{*} n\right)\right)$ if the model is Strong-CD or Receiver-CD. 


\subsection{Verifying the Correctness of an Estimate}

In this section, we show how to use a dense Census algorithm to verify whether a given estimate $\tilde{n}$ of network size is correct. Suppose that there are $n$ devices agreeing on a number $\tilde{n}$. We present an algorithm $\operatorname{Verify}(\tilde{n})$ that is able to check whether $\tilde{n}$ is a good estimate of $n$. We require that (i) a leader is elected if $n / 1.5 \leq \tilde{n} \leq 1.5 n$, and (ii) no leader is elected if $\tilde{n} \geq 1.9 n$ or $\tilde{n} \leq n / 1.9{ }^{4}$ The algorithm consists of two steps: The first step is to assign IDs in [N] to some devices, where $N=\Theta(\log \tilde{n})$. The second step is to check whether $\tilde{n}$ is a correct estimate via a dense Census algorithm on the ID space $[N]$ with a density parameter $c$ to be determined.

Step 1-ID Assignment. We first consider the case where sender-side collision detection is available (i.e., Strong-CD and Sender-CD). We initialize $S_{\text {bad }}=\emptyset$. The procedure of ID assignment consists of $N$ time slots. For each $i \in[N]$, at the $i$ th time slot each device $s \notin S_{\text {bad }}$ transmits a message with probability $1 / \tilde{n}$ to bid for the ID $i$. If a device $s$ hears back its message at the $i$ th time slot, then $s$ is the only one that transmits at the $i$ th time slot, and so we let $s$ assign itself the ID $i$.

We let $\beta$ be an upper limit on the number of times a device can transmit, where $\beta$ is a sufficiently large constant. The purpose of setting this limit is to ensure that the energy cost is low. For each device $s$, if $s$ has already transmitted for $\beta$ times during the first $i$ time slots, then we add $s$ to the set $S_{\mathrm{bad}}$ at the end of the $i$ th time slot, and $s$ is not allowed to transmit in future time slots during the ID assignment.

Next, we consider the case where sender-side collision detection is not available (i.e., Receiver$\mathrm{CD}$ and No-CD). In this case, a transmitter does not know whether it is the only one transmitting. To resolve this issue, we increase the number of time slots from $N$ to $2 N$.

Let $i \in[N]$. At the beginning of the $(2 i-1)$ th time slot, each device $s \notin S_{\text {bad }}$ joins the set $A_{i}$ with probability $1 / \tilde{n}$, and then each device $s \notin S_{\text {bad }} \cup A_{i}$ joins the set $B_{i}$ with probability $1 / \tilde{n}$.

We will assign the ID $i$ to a device $s$ if $s \in A_{i}$ and $\left|A_{i}\right|=\left|B_{i}\right|=1$. The following procedure allows each device $s \in A_{i}$ to test if $\left|A_{i}\right|=\left|B_{i}\right|=1$ : At the $(2 i-1)$ th time slot, all devices in $A_{i}$ transmit, and all devices in $B_{i}$ listen. At the (2i)th time slot, all devices in $B_{i}$ that have successfully received a message at the $(2 i-1)$ th time slot transmit, and all devices in $A_{i}$ listen. Notice that a device $s \in A_{i}$ successfully receives a message at the (2i)th time slot if and only if $\left|A_{i}\right|=\left|B_{i}\right|=1$.

Similarly, we set $\beta$ as an upper limit on the number of times a device can join the sets $A_{i}$ and $B_{i}, i \in[N]$. Any device $s$ that has already joined these sets for $\beta$ times is added to the set $S_{\text {bad. }}$.

Define $c=0.325$ if the model is Strong-CD or Sender-CD; otherwise, let $c=0.325^{2}$. The following lemma relates the density of the ID space to the accuracy of the estimate $\tilde{n}$ :

Lemma 17. Suppose that $\tilde{n} \geq 100$. With probability $1-\min \left\{n^{-\Omega(1)}, \tilde{n}^{-\Omega(1)}\right\}$, the following conditions are met: $(i)$ when $\tilde{n} \geq 1.9 n$ or $\tilde{n} \leq n / 1.9$, either $\left|S_{\text {bad }}\right|>0$ or the number of IDs that are assigned to devices is smaller than $c N$; (ii) when $n / 1.5 \leq \tilde{n} \leq 1.5 n$, we have $\left|S_{\text {bad }}\right|=0$ and the number IDs that are assigned to devices is higher than $c N$.

Proof. We write $\mathcal{A}$ to denote the ID assignment algorithm, and write $\mathcal{A}^{\prime}$ to denote a variant of the ID assignment algorithm that allows each device $s \in S_{\text {bad }}$ to continue participating (i.e., there is no upper limit about the number of transmission per device). The algorithm $\mathcal{A}^{\prime}$ is much easier to analyze than $\mathcal{A} .^{5}$ It is straightforward to see that in $\mathcal{A}^{\prime}$ the probability that an ID $i \in[N]$ is assigned is $\operatorname{Pr}[\operatorname{Binomial}(n, 1 / \tilde{n})=1](\operatorname{resp} ., \operatorname{Pr}[\operatorname{Binomial}(n, 1 / \tilde{n})=1] \cdot \operatorname{Pr}[\operatorname{Binomial}(n-1,1 / \tilde{n})=$ $1]$ ) when the model is Strong-CD or Sender-CD (resp., Receiver-CD or No-CD).

We only prove the lemma for the case where the model is Strong-CD or Sender-CD; the other case is similar. Observe that the following inequalities hold, given that $\tilde{n} \geq 100$. If $\tilde{n} \geq 1.9 n$ or

\footnotetext{
${ }^{4}$ In general, the constants 1.5 and 1.9 can both be made arbitrarily close to 1 , at the cost of more time and energy.

${ }^{5}$ In the analysis of $\mathcal{A}^{\prime}$, we still maintain the set $S_{\text {bad }}$, but the devices in $S_{\text {bad }}$ do not stop participating.
} 
$\tilde{n} \leq n / 1.9$, then

$$
\operatorname{Pr}[\operatorname{Binomial}(n, 1 / \tilde{n})=1]<0.32=c-0.005<c .
$$

If $n / 1.5 \leq \tilde{n} \leq 1.5 n$, then

$$
\operatorname{Pr}[\operatorname{Binomial}(n, 1 / \tilde{n})=1]>0.33=c+0.005>c .
$$

We use subscript to indicate whether a probability or an expected number refers to $\mathcal{A}$ or $\mathcal{A}^{\prime}$. For instance, given an event $A$, the notation $\operatorname{Pr}_{\mathcal{A}}[A]$ is the probability that $A$ occurs in an execution of $\mathcal{A}$. We write $X$ to denote the number of IDs in $[N]$ assigned to devices. We define $\mu$ as $\mathrm{E}_{\mathcal{A}^{\prime}}[X]=$ $N \operatorname{Pr}[\operatorname{Binomial}(n, 1 / \tilde{n})=1]$.

Case 1. Suppose $\tilde{n} \geq 1.9 n$. We need to prove that $\operatorname{Pr}_{\mathcal{A}}\left[\left|S_{\text {bad }}\right|=0 \wedge X \geq c N\right]=\tilde{n}^{-\Omega(1)}$. Observe that

$$
\operatorname{Pr}_{\mathcal{A}}\left[\left|S_{\text {bad }}\right|=0 \wedge X \geq c N\right]=\operatorname{Pr}_{\mathcal{P}^{\prime}}\left[\left|S_{\text {bad }}\right|=0 \wedge X \geq c N\right] \leq \operatorname{Pr}_{\mathcal{H}^{\prime}}[X \geq c N] .
$$

Thus, it suffices to show that $\operatorname{Pr}_{\mathcal{H}^{\prime}}[X \geq c N]=\tilde{n}^{-\Omega(1)}$. Let $\delta=\frac{c-\operatorname{Pr}[\operatorname{Binomial}(n, 1 / \tilde{n})=1]}{\operatorname{Pr}[\operatorname{Binomial}(n, 1 / \tilde{n})=1]}>0$. Then $\operatorname{Pr}_{\mathcal{H}^{\prime}}[X \geq c N]=\operatorname{Pr}_{\mathcal{H}^{\prime}}[X \geq(1+\delta) \mu]$. By a Chernoff bound, this is at most $\exp (-\delta \mu / 3)$ if $\delta>1$, and is at most $\exp \left(-\delta^{2} \mu / 3\right)$ if $\delta \leq 1$. Since $c-\operatorname{Pr}[\operatorname{Binomial}(n, 1 / \tilde{n})=1]>0.005$, we have: $\delta \mu=$ $(c-\operatorname{Pr}[\operatorname{Binomial}(n, 1 / \tilde{n})=1]) N \geq 0.005 N$ and $\delta^{2} \mu=\frac{(c-\operatorname{Pr}[\operatorname{Binomial}(n, 1 / \tilde{n})=1])^{2} N}{\operatorname{Pr}[\operatorname{Binomial}(n, 1 / \tilde{n})=1]} \geq 0.005^{2} N$. Thus, $\operatorname{Pr}_{\mathcal{A}^{\prime}}[X \geq c N]=\exp (-\Omega(N))=\tilde{n}^{-\Omega(1)}$.

Case 2. Suppose $n / 1.5 \leq \tilde{n} \leq 1.5 n$. We need to prove that $\operatorname{Pr}_{\mathcal{A}}\left[\left|S_{\text {bad }}\right|>0 \vee X \leq c N\right]=\tilde{n}^{-\Omega(1)}$. Observe that

$$
\begin{aligned}
\operatorname{Pr}_{\mathcal{A}}\left[\left|S_{\mathrm{bad}}\right|>0 \vee X \leq c N\right] & \leq \operatorname{Pr}_{\mathcal{A}}\left[\left|S_{\mathrm{bad}}\right|>0\right]+\operatorname{Pr}_{\mathcal{A}}\left[X \leq c N \wedge\left|S_{\mathrm{bad}}\right|=0\right] \\
& \leq \operatorname{Pr}_{\mathcal{H}^{\prime}}\left[\left|S_{\mathrm{bad}}\right|>0\right]+\operatorname{Pr}_{\mathcal{H}^{\prime}}\left[X \leq c N \wedge\left|S_{\mathrm{bad}}\right|=0\right] \\
& \leq \operatorname{Pr}_{\mathcal{H}^{\prime}}\left[\left|S_{\mathrm{bad}}\right|>0\right]+\operatorname{Pr}_{\mathcal{H}^{\prime}}[X \leq c N] .
\end{aligned}
$$

Thus, it suffices to show that both $\operatorname{Pr}_{\mathcal{H}^{\prime}}[X \leq c N]$ and $\operatorname{Pr}_{\mathcal{A}^{\prime}}\left[\left|S_{\text {bad }}\right|>0\right]$ are upper bounded by $\tilde{n}^{-\Omega(1)}$. Let $\delta=\frac{\operatorname{Pr}[\operatorname{Binomial}(n, 1 / \tilde{n})=1]-c}{\operatorname{Pr}[\operatorname{Binomial}(n, 1 / \tilde{n})=1]}>0$. Then $\operatorname{Pr}_{\mathcal{H}^{\prime}}[X \leq c N]=\operatorname{Pr}_{\mathcal{H}^{\prime}}[X \leq(1-\delta) \mu]$. By a Chernoff bound, this is at most $\exp (-\delta \mu / 3)$ if $\delta>1$, and is at most $\exp \left(-\delta^{2} \mu / 2\right)$ if $\delta \leq 1$. Similarly, $\operatorname{Pr}[\operatorname{Binomial}(n, 1 / \tilde{n})=1]-c>0.005$, and hence $\operatorname{Pr}_{\mathcal{H}^{\prime}}[X \leq c N]=\exp (-\Omega(N))=\tilde{n}^{-\Omega(1)}$.

Next, we calculate $\operatorname{Pr}_{\mathcal{H}^{\prime}}\left[\left|S_{\text {bad }}\right|>0\right]$. The probability that a device $s$ joins $S_{\text {bad }}$ in $\mathcal{A}^{\prime}$ is

$$
\operatorname{Pr}[\operatorname{Binomial}(N, 1 / \tilde{n}) \geq \beta] \leq N^{\beta} \tilde{n}^{-\beta} \text {. }
$$

By a union bound over all $n$ devices, $\operatorname{Pr}_{\mathcal{H}^{\prime}}\left[\left|S_{\text {bad }}\right|>0\right] \leq N^{\beta} \tilde{n}^{-\beta} n=\tilde{n}^{-\Omega(1)}$, since $N=\Theta(\log (\tilde{n}))$, $n / 1.5 \leq \tilde{n} \leq 1.5 n$, and $\beta=\Omega(1)$.

Case 3. Suppose $\tilde{n} \leq n / 1.9$. We need to prove that $\operatorname{Pr}_{\mathcal{A}}\left[\left|S_{\text {bad }}\right|=0 \wedge X \geq c N\right]=n^{-\Omega(1)}$. Similar to the first case, it suffices to show that $\operatorname{Pr}_{\mathcal{H}^{\prime}}[X \geq c N]=n^{-\Omega(1)}$. Notice that the same calculation for the first case can be applied here, and so we already have $\operatorname{Pr}_{\mathcal{A}^{\prime}}[X \geq c N]=\tilde{n}^{-\Omega(1)}$. Thus, we only need to focus on the situation where $n$ is significantly larger than $\tilde{n}$.

Assuming $n \geq \tilde{n}^{2}$, we have

$$
\begin{aligned}
\operatorname{Pr}_{\mathcal{A}^{\prime}}[X>0] & \leq N \operatorname{Pr}[\operatorname{Binomial}(n, 1 / \tilde{n})=1] \\
& \leq N(n / \tilde{n})(1-1 / \tilde{n})^{n-1} \\
& =\exp (-\Omega(n / \tilde{n})) \\
& =\exp (-\Omega(\sqrt{n})) .
\end{aligned}
$$

Step 2-Checking the Correctness of Estimate.. We run a dense Census algorithm with ID space $[N]$ and parameter $c$. It is possible that a device is assigned to multiple IDs, and in such case the 
device simulates multiple devices of different IDs in the dense Census algorithm. If the number of IDs that are assigned is at least $c N$, then after solving Census, all devices that are assigned IDs know the list of all IDs in $[N]$ that are assigned to devices.

We first consider the case where sender-side collision detection is available. Let $s_{1}$ be the device that has the smallest ID. We allocate a special time slot $t^{*}$, where $s_{1}$ and all devices in $S_{\text {bad }}$ transmit. The device $s_{1}$ elects itself as the leader if (i) $s$ has collected a list of IDs of size at least $c N$ during the Census algorithm (i.e., the number of IDs that are assigned to devices is at least $c N$ ), and (ii) $s$ is able to hear back its message at time $t^{*}$, i.e., $S_{\text {bad }}=\emptyset$.

For the case where sender-side collision detection is not available, $s_{1}$ cannot simultaneously transmit and listen. To solve this issue, we let $s_{2}$ be the device that holds the smallest ID in $[N]$ excluding the ones assigned to $s_{1}$. Notice that a device can be assigned at most $\beta$ IDs. We let $s_{2}$ listen to the time slot $t^{*}$, and $s_{2}$ elects itself as the leader if $s_{2}$ hears a message from $s_{1}$ and the ID list resulting from the Census algorithm has size at least $c N$.

The correctness of Verify $(\tilde{n})$ follows from Lemma 18. The first step costs $O(N)=O(\log \tilde{n})$ time and $O(\beta)=O(1)$ energy. The second step costs $O(N)=O(\log \tilde{n})$ time and $O(\alpha(N))=O(\alpha(\tilde{n}))$ energy. We conclude the following lemma:

LEMMA 18. With probability $1-\min \left\{n^{-\Omega(1)}, \tilde{n}^{-\Omega(1)}\right\}$, the algorithm Verify $(\tilde{n})$ accomplishes the following in time $O(\log \tilde{n})$ with energy $O(\alpha(\tilde{n}))$. A leader is elected if $n / 1.5 \leq \tilde{n} \leq 1.5 n$, and no leader is elected if $\tilde{n} \geq 1.9 n$ or $\tilde{n} \leq n / 1.9$.

The asymptotic time complexity of the algorithm $\operatorname{Verify}(\tilde{n})$ is the same as the algorithm in Reference [7], which works in Strong-CD and is based on circuit simulation. However, the circuit simulation takes only $O(1)$ energy while Verify $(\tilde{n})$ needs $O(\alpha(\tilde{n}))$ energy.

\subsection{Exponential Search}

Let $D=\left\{d_{1}, d_{2}, \ldots\right\}$ be an infinite set of positive integers such that $d_{i+1} \geq \gamma \cdot d_{i}$ for each $i \geq 1$, where $\gamma>1$ is some large enough constant. We define $\hat{i}$ as the index such that $d_{\hat{i}-1}<\log n \leq$ $d_{\hat{i}}$, where $n$ is the network size. For the Strong-CD and the Receiver-CD models, we present an algorithm $\operatorname{ExpSearch}(D)$ that estimates $\hat{i}$ within a \pm 1 additive error in $O(\log \hat{i})$ time.

We first define a 1-round subroutine Test $(i)$ as follows: Each device transmits a message with probability $2^{-d_{i}}$, and all other devices listen to the channel. For each listener $s$, it decides " $i \geq$ $\hat{i}$ " if the channel is silent, and it decides " $i<\hat{i}$ " otherwise. Each transmitter decides " $i<\hat{i}$." It is straightforward to see that all devices make the same decision. We have the following lemma:

LEMma 19. Consider an execution of Test $(i)$. The following holds with probability $1-n^{-\Omega(1)}$. If $i \leq \hat{i}-2$, then all devices decide " $i<\hat{i}$." If $i \geq \hat{i}+1$, then all devices decide " $i \geq \hat{i}$."

Proof. Recall that $\gamma=\Omega(1)$ is chosen to be sufficiently large. For any $i \leq \hat{i}-2$, the probability that $\operatorname{Test}(i)$ returns " $i \geq \hat{i}$ " is $\operatorname{Pr}\left[\operatorname{Binomial}\left(n, 2^{-d_{i}}\right)=0\right]=n\left(1-2^{-d_{i}}\right)^{n} \leq n\left(1-2^{\frac{\log n}{\gamma}}\right)^{n}=$ $n \cdot\left(1-n^{-1 / \gamma}\right)^{n} \leq n \cdot \exp \left(-n^{1-1 / \gamma}\right)=n^{\Omega(1)}$. For any $i \geq \hat{i}+1$, the probability that Test $(i)$ returns " $i<\hat{i}$ " is $\operatorname{Pr}\left[\operatorname{Binomial}\left(n, 2^{-d_{i}}\right)>0\right] \leq n \cdot 2^{-d_{i}} \leq n \cdot 2^{-\gamma \log n}=n^{-\gamma+1}=n^{\Omega(1)}$.

Based on the subroutine Test $(i)$, the procedure $\operatorname{ExpSearch}(D)$ is defined as follows: The first step is to repeatedly run Test $(i)$ for $i=1,2,4,8, \ldots$ until we reach the first index $i^{\prime}$ such that all devices decide " $i$ ' $\geq \hat{i}$ " during Test $\left(i^{\prime}\right)$. Then, we conduct a binary search using Test $(i)$ on the set $\left\{1,2,3, \ldots, i^{\prime}\right\}$ to find the smallest index $i$ such that Test $(i)$ returns " $i \geq \hat{i}$." Due to Lemma 19 , if all Test $(i)$ do not fail, then it is clear that such an index $\tilde{i}$ satisfies that $\tilde{i} \in\{\hat{i}-1, \hat{i}, \hat{i}+1\}$. We conclude the following lemma: 
LEMmA 20. In the Strong-CD and the Receiver-CD models, the algorithm ExpSearch $(D)$ finds an index $\tilde{i}$ such that $\tilde{i} \in\{\hat{i}-1, \hat{i}, \hat{i}+1\}$ in $O(\log \hat{i})$ time with probability $1-n^{-\Omega(1)}$.

\subsection{Main Algorithm}

In this section, we prove Theorem 7. We will present an algorithm that finds an estimate $\tilde{n}$ that is within a factor of 2 of the network size $n$, i.e., $n / 2 \leq \tilde{n} \leq 2 n$. Our algorithm EstimateSize(D) takes an infinite set $D=\left\{d_{1}, d_{2}, \ldots\right\}$ of positive integers as an input parameter. We require that $d_{i+1} \geq \gamma d_{i}$ and $d_{1}$ is sufficiently large such that $\sum_{k=d_{1}}^{\infty} 1 / \sqrt{2^{k}} \leq 1$ and $\sqrt{2^{d_{1}}} \geq 100$. We will later see that different choices of $D$ lead to different time-energy tradeoffs.

With respect to the set $D$, define $\hat{i}$ as the index such that $d_{\hat{i}-1}<\log n \leq d_{\hat{i}}$. The elements in the set $D$ play the roles of "checkpoints" in our algorithm. The set $D$ is independent of $n$, but $\hat{i}$ is a function of $n$. In subsequent discussion, we assume $n>2^{d_{1}}$, and so the index $\hat{i}$ is well defined. The reason that we are allowed to make this assumption is that for the case where $n \leq 2^{d_{1}}=O(1)$, we can run any Approximate Counting algorithm to find an estimate of $n$ in $O(1)$ time. The algorithm EstimateSize(D) is as follows:

Initial Setup. For each integer $k \geq d_{1}$, a device $s$ is labeled $k$ with probability $1 / \sqrt{2^{k}}$ in such a way that $s$ is labeled by at most one number; this is the reason that we require $\sum_{k=d_{1}}^{\infty} 1 / \sqrt{2^{k}} \leq 1$. We write $S_{k}$ to denote the set of all devices labeled $k$. For the case that the model is Strong-CD or Receiver-CD, we do $\operatorname{ExpSearch}(D)$, and let $\tilde{i}$ be the result of $\operatorname{ExpSearch}(D)$, and set $k_{0}=d_{\tilde{i}-2}$. For the case that the model is Sender-CD or No-CD, set $k_{0}=d_{1}$.

Finding an Estimate. For $k=k_{0}, k_{0}+1, k_{0}+2, \ldots$, do the following task: The devices in $S_{k}$ collaboratively run Verify $\left(\sqrt{2^{k}}\right)$. For the special case that a checkpoint is met, i.e., $k=d_{i}$ for some $i$, do the following additional task: Let $L_{e}$ (resp., $L_{o}$ ) be the set of leaders elected in Verify $\left(\sqrt{2^{k^{\prime}}}\right)$ for all even (resp., odd) $k^{\prime}$ so far (i.e., $k^{\prime} \in\left[k_{0}, k\right]$ ). We let all devices in $L_{o}$ simultaneously announce their labels, while all other devices listen. If exactly one message $\tilde{k}$ is sent, the algorithm is terminated with all devices agreeing on the same estimate $\tilde{n}=2^{\tilde{k}}$. If the algorithm has not terminated yet, repeat the above with $L_{e}$.

Lemma 21. Define $\hat{k}=\lceil\log n\rceil$. With probability $1-\exp (\Omega(\sqrt{n}))$, the following holds. For each $k \in[1, \hat{k}-2]$, we have $\left|S_{k}\right| \geq 1.9 \sqrt{n / 2} \geq 1.9 \sqrt{2^{k}}$. For each $k \in[\hat{k}+1, \infty)$, we have $\left|S_{k}\right| \leq \sqrt{2 n} / 1.9 \leq$ $\sqrt{2^{k}} / 1$.9. For at least one of $k \in\{\hat{k}-1, \hat{k}\}$, we have $\sqrt{2^{k}} / 1.5 \leq\left|S_{k}\right| \leq 1.5 \sqrt{2^{k}}$.

Proof. First, with probability $1-n \cdot \sum_{k=n+1}^{\infty} 1 / \sqrt{2^{k}}=1-\exp (-\Omega(n))$, no device has label greater than $n$. Therefore, in what follows, we only consider the labels in the range $\{1,2, \ldots, n\}$.

Consider the case $k \leq \hat{k}-2$. We have $\mu=\mathrm{E}\left[\left|S_{k}\right|\right]=n \cdot \sqrt{2^{-k}} \geq n \cdot \sqrt{2^{-(\log n-1)}}=\sqrt{2 n}$. Using a Chernoff bound with $\delta=0.05$, the probability that $\left|S_{k}\right| \leq 1.9 \sqrt{2^{k}} \leq 1.9 \sqrt{n / 2} \leq(1-\delta) \mu$ can be upper bounded by $\exp \left(-\delta^{2} \mu / 2\right)=\exp (-\Omega(\sqrt{n}))$.

Consider the case $n \geq k \geq \hat{k}+1$. We have $\mu=\mathrm{E}\left[\left|S_{k}\right|\right]=n \cdot \sqrt{2^{-k}} \leq n \cdot \sqrt{2^{-(\log n+1)}}=\sqrt{n / 2}$. Using a Chernoff bound with $\delta=1 / 1.9$, the probability that $\left|S_{k}\right| \geq \sqrt{2^{k}} / 1.9 \geq \sqrt{2 n} / 1.9 \geq(1+\delta) \mu$ can be upper bounded by $\exp \left(-\delta^{2} \mu / 3\right)=\exp (-\Omega(\sqrt{n}))$.

Among the two numbers in $\{\hat{k}-1, \hat{k}\}$, we select $k \in\{\hat{k}-1, \hat{k}\}$ such that $n / \sqrt{2} \leq 2^{k} \leq \sqrt{2} n$. Then the expected number $\mu=\mathrm{E}\left[\left|S_{k}\right|\right]$ satisfies $\sqrt{2^{k}} / 1.5<\sqrt{2^{k}} / \sqrt{2} \leq \mu \leq \sqrt{2} \cdot \sqrt{2^{k}}<1.5 \sqrt{2^{k}}$. Similarly, using a Chernoff bound, we can infer that the probability that $\left|S_{k}\right|$ is not within $\sqrt{2^{k}} / 1.5$ and $1.5 \sqrt{2^{k}}$ is at most $\exp (-\Omega(\sqrt{n}))$.

LEMMA 22. In an execution of EstimateSize(D), with probability $1-n^{-\Omega(1)}$, none of Verify $\left(\sqrt{2^{k}}\right)$ fails. 
Proof. We assume that the statement of Lemma 21 holds, since it holds with probability $1-\exp (\Omega(\sqrt{n}))$. Similarly, with probability $1-n \cdot \sum_{k=n+1}^{\infty} 1 / \sqrt{2^{k}}=1-\exp (-\Omega(n))$, no device has label greater than $n$. Therefore, in what follows, we only consider the labels in the range $\{1,2, \ldots, n\}$.

By Lemma 18, the failure probability of Verify $\left(\sqrt{2^{k}}\right)$ is at most $\min \left\{{\sqrt{2^{k}}}^{-\Omega(1)},\left|S_{k}\right|^{-\Omega(1)}\right\}$. Define $\hat{k}=\lceil\log n\rceil$. If $k \geq \hat{k}+1$, then the failure probability of $\operatorname{Verify}\left(\sqrt{2^{k}}\right)$ is at most $\sqrt{2^{k}}-\Omega(1)=n^{-\Omega(1)}$. By Lemma 21, if $k \leq \hat{k}$, then $\left|S_{k}\right|=\Omega(\sqrt{n})$, and so the failure probability of Verify $\left(\sqrt{2^{k}}\right)$ is at most $\left|S_{k}\right|^{-\Omega(1)}=n^{-\Omega(1)}$. By a union bound over all $k \in\{1, \ldots, n\}$, the probability that at least one of $\operatorname{Verify}\left(\sqrt{2^{k}}\right)$ fails is bounded by $n \cdot n^{-\Omega(1)}=n^{-\Omega(1)}$.

LEMMA 23. In an execution of EstimateSize(D), with probability $1-n^{-\Omega(1)}$, all devices agree on an estimate $\tilde{n}$ such that $n / 2 \leq \tilde{n} \leq 2 n$ in time $T(n)=O\left(d_{\hat{i}}^{2}\right)$ with energy cost $E(n)$, where $E(n)=O(\log \hat{i})$ in Strong-CD and Receiver-CD, and $E(n)=O(\hat{i})$ in Sender-CD and No-CD.

Proof. We assume that all of $\operatorname{ExpSearch}(D)$ and $\operatorname{Verify}\left(\sqrt{2^{k}}\right)$, for all $k$, do not fail, since the probability that at least one of them fails is $n^{-\Omega(1)}$, in view of Lemma 20 and Lemma 22 . We also assume that the statement of Lemma 21 holds, since it holds with probability $1-\exp (\Omega(\sqrt{n})$ ).

Define $\hat{k}=\lceil\log n\rceil$. A consequence of Lemma 21 is that (i) there exists $k \in\{\hat{k}-1, \hat{k}\}$ such that Verify $\left(\sqrt{2^{k}}\right)$ elects a leader, and (ii) for each $k \notin\{\hat{k}-1, \hat{k}\}$, Verify $\left(\sqrt{2^{k}}\right)$ does not elect a leader. Recall that $\hat{i}$ is defined as the index $i$ such that $d_{i-1}<\log n \leq d_{i}$, and so the algorithm EstimateSize(D) must end by the iteration $k=d_{\hat{i}}$ with a correct estimate of $n$.

In what follows, we analyze the runtime and the energy cost of EstimateSize(D). Since each $\operatorname{Verify}\left(\sqrt{2^{k}}\right)$ takes $O(k)$ time, the total time complexity is $d_{\hat{i}} \cdot O\left(d_{\hat{i}}\right)=O\left(d_{\hat{i}}^{2}\right)$.

The energy cost per device in $S_{k}$ to make the call Verify $\left(\sqrt{2^{k}}\right)$ is $O\left(\alpha\left(\left|S_{k}\right|\right)\right)=O(\alpha(n))$, which will never be the dominant cost. In Sender-CD and No-CD, the asymptotic energy cost of EstimateSize(D) equals the number of times we encounter a checkpoint $k=d_{i}$ for some $d_{i} \in D$, which is $O(\hat{i})$.

Next, we analyze the energy cost in Strong-CD and Receiver-CD. Due to ExpSearch $(D)$ during the initial setup, the number of checkpoints encountered is reduced to $O(1)$, as we start with $k_{0}=d_{\tilde{i}-2}$, where the index $\tilde{i}$ is the result of $\operatorname{ExpSearch}(D)$ and satisfies $\tilde{i} \in\{\hat{i}-1, \hat{i}, \hat{i}+1\}$. Therefore, the asymptotic energy cost of EstimateSize(D) equals the energy cost of ExpSearch $(D)$, which is $O(\log \hat{i})$.

In addition to solving Approximate Counting, the algorithm EstimateSize(D) also solves Leader Election. Notice that by the end of EstimateSize(D), a unique device $s$ announces its label while all other devices listen to the channel.

Setting the Checkpoints. Lemma 23 naturally offers a time-energy tradeoff. We demonstrate how different choices of the checkpoints $D$ give rise to different runtime and energy cost specified in Table 1. For the base case, the first checkpoint $d_{1}$ is always chosen as a large enough constant to meet the three conditions: $d_{i+1} \geq \gamma d_{i}, \sum_{k=d_{1}}^{\infty} 1 / \sqrt{2^{k}} \leq 1$, and $\sqrt{2^{d_{1}}} \geq 100$. In subsequent discussion, we only focus on how we define $d_{i}$ inductively.

To obtain $O\left(\log ^{2} n\right)$ runtime, we set $d_{i}=\gamma d_{i-1}$ for some constant $\gamma$. Recall that $\hat{i}$ is defined as the index $i$ such that $d_{i-1}<\log n \leq d_{i}$, and so $d_{\hat{i}} \leq \gamma \log n$. Thus, the runtime is $O\left(d_{\hat{i}}^{2}\right)=O\left(\log ^{2} n\right)$. With such checkpoints, the energy cost in Sender-CD and No-CD is $O(\hat{i})=O(\log \log n)$; the energy cost in Strong-CD and Receiver-CD is $O(\log \hat{i})=O(\log \log \log n)$.

For $0<\epsilon \leq O(1)$. To obtain $O\left(\log ^{2+\epsilon} n\right)$ runtime, we set $d_{i}=d_{i-1}^{1+\epsilon / 2}$. Notice that $d_{\hat{i}} \leq \log ^{1+\epsilon / 2} n$. Thus, the runtime is $O\left(d_{\hat{i}}^{2}\right)=O\left(\log ^{2+\epsilon} n\right)$. With such checkpoints, the energy cost in Sender-CD 
and No-CD is $O(\hat{i})=O\left(\log _{1+\epsilon / 2} \log \log n\right)=O\left(\epsilon^{-1} \log \log \log n\right)$; the energy cost in Strong-CD and Receiver-CD is $O(\log \hat{i})=O\left(\log \left(\epsilon^{-1} \log \log \log n\right)\right)$.

Theorem 7 is proved as follows: Setting $d_{i}=b^{d_{i-1}}$ for any constant $b>1$ yields a polynomial time algorithm achieving the desired energy complexity, as $O(\hat{i})=O\left(\log ^{*} n\right)$ and $O(\log \hat{i})=$ $O\left(\log \log ^{*} n\right)$. To obtain $n^{o(1)}$ runtime while maintaining the same asymptotic energy complexity, we can use $d_{i}=2^{2^{\left(\log d_{i-1}\right)^{\epsilon}}}$, for some constant $0<\epsilon<1$. Since $\hat{i}$ is chosen such that $d_{\hat{i}-1}<\log n$, we have $d_{\hat{i}} \leq 2^{2^{(\log \log n)^{\epsilon}}}$, and so the runtime is $O\left(d_{\hat{i}}^{2}\right)=O\left(2^{2^{1+(\log \log n)^{\epsilon}}}\right)=n^{o(1)}$.

\section{CONCLUSION AND OPEN PROBLEMS}

In this article we exposed two exponential separations in the energy complexity of Leader Election on various wireless radio network models. The upshot is that randomized algorithms in \{Strong$C D$, Receiver-CD $\}$ are exponentially more efficient than those in $\{$ Sender-CD, No-CD $\}$, but deterministic algorithms in $\{$ Strong-CD,Sender-CD $\}$ are exponentially more efficient than those in $\{$ Receiver-CD,No-CD\}. This exponential separation also occurs in the closely related problem of Approximate Counting.

There are a few intriguing problems that remain open in the context of single-hop networks. For example, is $\Theta(\alpha(N))$ the correct complexity of Leader Election and Census for dense instances? What is the true complexity of Approximate Counting? In general, it should exhibit a three-way tradeoff between energy, time, and a given error probability. Can $n$ anonymous devices assign themselves IDs in $\{1, \ldots, n\}$ with $o(\log \log n)$ energy [40] in the worst case?

Little is known about the energy-complexity of fundamental graph problems in arbitrary (multihop) networks. Recently, Chang et al. [10] studied the energy complexity for broadcasting in multihop networks. It is an interesting future work direction to investigate the energy complexity for other fundamental graph problems.

\section{REFERENCES}

[1] N. Alon, A. Bar-Noy, N. Linial, and D. Peleg. 1991. A lower bound for radio broadcast. 7. Comput. System Sci. 43, 2 (1991), 290-298.

[2] R. Bar-Yehuda, O. Goldreich, and A. Itai. 1991. Efficient emulation of single-hop radio network with collision detection on multi-hop radio network with no collision detection. Distrib. Comput. 5, 2 (1991), 67-71.

[3] R. Bar-Yehuda, O. Goldreich, and A. Itai. 1992. On the time-complexity of broadcast in multi-hop radio networks: An exponential gap between determinism and randomization. J. Comput. System Sci. 45, 1 (1992), 104-126.

[4] M. Barnes, C. Conway, J. Mathews, and D. K. Arvind. 2010. ENS: An energy harvesting wireless sensor network platform. In Proceedings of the 5th International Conference on Systems and Networks Communications. 83-87. DOI : https://doi.org/10.1109/ICSNC.2010.18

[5] M. A. Bender, J. T. Fineman, S. Gilbert, and M. Young. 2016. How to scale exponential backoff: Constant throughput, polylog access attempts, and robustness. In Proceedings of the 27th ACM-SIAM Symposium on Discrete Algorithms (SODA'16). 636-654. DOI : https://doi.org/10.1137/1.9781611974331.ch47

[6] M. A. Bender, J. T. Fineman, M. Movahedi, J. Saia, V. Dani, S. Gilbert, S. Pettie, and M. Young. 2015. Resourcecompetitive algorithms. SIGACT News 46, 3 (2015), 57-71. DOI: https://doi.org/10.1145/2818936.2818949

[7] M. A. Bender, T. Kopelowitz, S. Pettie, and M. Young. 2016 (also to appear in SIAM fournal on Computing). Contention resolution with log-logstar channel accesses. In Proceedings of the 48th ACM Symposium on Theory of Computing (STOC'16). 499-508. DOI : https://doi.org/10.1145/2897518.2897655

[8] P. Brandes, M. Kardas, M. Klonowski, D. Pajak, and R. Wattenhofer. 2016. Approximating the size of a radio network in beeping model. In Proceedings of the 23rd International Colloquium on Structural Information and Communication Complexity (SIROCCO'16). 358-373.

[9] K. Censor-Hillel, B. Haeupler, D. E. Hershkowitz, and G. Zuzic. 2017. Broadcasting in noisy radio networks. In Proceedings of the ACM Symposium on Principles of Distributed Computing (PODC'17). 33-42. DOI : https://doi.org/10.1145/ 3087801.3087808 
[10] Y.-J. Chang, V. Dani, T. P. Hayes, Q. He, W. Li, and S. Pettie. 2018. The energy complexity of broadcast. In Proceedings of the ACM Symposium on Principles of Distributed Computing (PODC'18). ACM, New York, NY, 95-104. DOI : https:// doi.org/10.1145/3212734.3212774

[11] Y.-J. Chang, T. Kopelowitz, S. Pettie, R. Wang, and W. Zhan. 2017. Exponential separations in the energy complexity of leader election. In Proceedings of the 49th ACM SIGACT Symposium on Theory of Computing (STOC'17). ACM, New York, NY, 771-783. DOI : https://doi.org/10.1145/3055399.3055481

[12] B. S. Chlebus, D. R. Kowalski, and A. Pelc. 2012. Electing a leader in multi-hop radio networks. In Proceedings of the 16th International Conference on Principles of Distributed Systems (OPODIS'12). Springer, 106-120.

[13] A. E. F. Clementi, A. Monti, and R. Silvestri. 2003. Distributed broadcast in radio networks of unknown topology. Theoret. Comput. Sci. 302, 1 (2003), 337-364.

[14] A. Cornejo and F. Kuhn. 2010. Deploying wireless networks with beeps. In Proceedings of the 24th International Symposium on Distributed Computing (DISC'10). Springer, 148-162.

[15] A. Czumaj and P. Davies. 2016. Brief announcement: Optimal leader election in multi-hop radio networks. In Proceedings of the 35th ACM Symposium on Principles of Distributed Computing (PODC'16). 47-49. DOI : https://doi.org/ 10.1145/2933057.2933076

[16] A. Czumaj and P. Davies. 2017. Exploiting spontaneous transmissions for broadcasting and leader election in radio networks. In Proceedings of the ACM Symposium on Principles of Distributed Computing (PODC'17). 3-12. DOI : https:// doi.org/10.1145/3087801.3087825

[17] A. Czumaj and W. Rytter. 2003. Broadcasting algorithms in radio networks with unknown topology. In Proceedings of the 44th IEEE Symposium on Foundations of Computer Science (FOCS'03). 492-501.

[18] S. Daum, S. Gilbert, F. Kuhn, and C. Newport. 2012. Leader election in shared spectrum radio networks. In Proceedings of the 31st ACM Symposium on Principles of Distributed Computing (PODC'12). 215-224.

[19] P. Erdős, A. Rényi, and V. T. Sós. 1966. On a problem of graph theory. Studia Sci. Math. Hung. 1 (1966), $215-235$.

[20] M. Farach-Colton, R. J. Fernandes, and M. A. Mosteiro. 2006. Lower bounds for clear transmissions in radio networks. In Proceedings of the 7th Latin American Symposium on Theoretical Informatics (LATIN'06). 447-454. DOI : https://doi. org/10.1007/11682462_42

[21] M. Ghaffari and B. Haeupler. 2013. Near optimal leader election in multi-hop radio networks. In Proceedings of the 24th ACM-SIAM Symposium on Discrete Algorithms (PODC'13). 748-766.

[22] M. Ghaffari, N. A. Lynch, and S. Sastry. 2012. Leader election using loneliness detection. Distrib. Comput. 25, 6 (2012), 427-450. DOI : https://doi.org/10.1007/s00446-012-0172-x

[23] M. Ghaffari and C. Newport. 2016. Leader election in unreliable radio networks. In Proceedings of the 43rd International Colloquium on Automata, Languages, and Programming (ICALP'16) (Leibniz International Proceedings in Informatics (LIPIcs)), Vol. 55. Schloss Dagstuhl-Leibniz-Zentrum für Informatik, Dagstuhl, Germany, 138:1-138:14. DOI : https:// doi.org/10.4230/LIPIcs.ICALP.2016.138

[24] S. Gilbert, V. King, S. Pettie, E. Porat, J. Saia, and M. Young. 2014. (Near) optimal resource-competitive broadcast with jamming. In Proceedings of the 26th ACM Symposium on Parallelism in Algorithms and Architectures (SPAA'14). 257-266. DOI : https://doi.org/10.1145/2612669.2612679

[25] S. Gilbert and C. Newport. 2015. The computational power of beeps. In Proceedings of the 29th International Symposium on Distributed Computing (DISC'15). Springer, 31-46.

[26] A. G. Greenberg and S. Winograd. 1985. A lower bound on the time needed in the worst case to resolve conflicts deterministically in multiple access channels. J. ACM 32, 3 (1985), 589-596.

[27] T. Jurdzinski, M. Kutylowski, and J. Zatopianski. 2002. Efficient algorithms for leader election in radio networks. In Proceedings of the 21st ACM Symposium on Principles of Distributed Computing (PODC'02). 51-57. DOI : https://doi.org/ 10.1145/571825.571833

[28] T. Jurdzinski, M. Kutylowski, and J. Zatopianski. 2002. Energy-efficient size approximation of radio networks with no collision detection. In Proceedings of the 8th International Conference on Computing and Combinatorics (COCOON'02). 279-289. DOI : https://doi.org/10.1007/3-540-45655-4_31

[29] T. Jurdzinski, M. Kutylowski, and J. Zatopianski. 2002. Weak communication in radio networks. In Proceedings of the 8th International European Conference on Parallel Computing (Euro-Par'02). 965-972. DOI : https://doi.org/10.1007/ 3-540-45706-2_137

[30] T. Jurdzinski, M. Kutylowski, and J. Zatopianski. 2003. Weak communication in single-hop radio networks: adjusting algorithms to industrial standards. Concurr. Comput.: Pract. Exper. 15, 11-12 (2003), 1117-1131. DOI : https://doi.org/ $10.1002 /$ cpe.783

[31] T. Jurdzinski and G. Stachowiak. 2002. Probabilistic algorithms for the wakeup problem in single-hop radio networks. In Proceedings of the 13th International Symposium on Algorithms and Computation (ISAAC'02). 535-549. DOI : https:// doi.org/10.1007/3-540-36136-7_47 
[32] M. Kardas, M. Klonowski, and D. Pajak. 2013. Energy-efficient leader election protocols for single-hop radio networks. In Proceedings of the 42nd International Conference on Parallel Processing. 399-408.

[33] G. Katona and E. Szemerédi. 1967. On a problem of graph theory. Studia Scient. Math. Hung. 2 (1967), 23-28.

[34] V. King, J. Saia, and M. Young. 2011. Conflict on a communication channel. In Proceedings of the 30th ACM Symposium on Principles of Distributed Computing (PODC'11). 277-286. DOI : https://doi.org/10.1145/1993806.1993855

[35] D. R. Kowalski and A. Pelc. 2005. Broadcasting in undirected ad hoc radio networks. Distrib. Comput. 18, 1 (2005), 43-57. DOI : https://doi.org/10.1007/s00446-005-0126-7

[36] D. R. Kowalski and A. Pelc. 2009. Leader election in ad hoc radio networks: A keen ear helps. In Proceedings of the 36th International Colloquium on Automata, Languages and Programming (ICALP'09). 521-533. DOI : https://doi.org/ 10.1007/978-3-642-02930-1_43

[37] E. Kushilevitz and Y. Mansour. 1998. An $\Omega(D \log (N / D))$ lower bound for broadcast in radio networks. SIAM $\mathcal{f}$ Comput. 27, 3 (1998), 702-712. DOI : https://doi.org/10.1137/S0097539794279109

[38] M. Kutyłowski and W. Rutkowski. 2003. Adversary immune leader election in ad hoc radio networks. In Proceedings of the 11th European Symposium on Algorithms (ESA'03). Springer, 397-408.

[39] Y. Lee, S. Bang, I. Lee, Y. Kim, G. Kim, M. H. Ghaed, P. Pannuto, P. Dutta, D. Sylvester, and D. Blaauw. 2013. A modular $1 \mathrm{~mm}^{3}$ die-stacked sensing platform with low power $\mathrm{I}^{2} \mathrm{C}$ inter-die communication and multi-modal energy harvesting. IEEE J. Solid-State Circ. 48, 1 (2013), 229-243.

[40] K. Nakano and S. Olariu. 2000. Energy-efficient initialization protocols for single-hop radio networks with no collision detection. IEEE Trans. Parallel Distrib. Syst. 11, 8 (2000), 851-863. DOI : https://doi.org/10.1109/71.877942

[41] K. Nakano and S. Olariu. 2000. Randomized initialization protocols for ad hoc networks. IEEE Trans. Parallel Distrib. Syst. 11, 7 (2000), 749-759. DOI : https://doi.org/10.1109/71.877833

[42] C. C. Newport. 2014. Radio network lower bounds made easy. In Proceedings of the 28th International Symposium on Distributed Computing (DISC'14). 258-272. DOI : https://doi.org/10.1007/978-3-662-45174-8_18

[43] J. Polastre, R. Szewczyk, and D. Culler. 2005. Telos: Enabling ultra-low power wireless research. In Proceedings of the 4th International Symposium on Information Processing in Sensor Networks (IPSN'05). 364-369. DOI : https://doi.org/10. 1109/IPSN.2005.1440950

[44] J. Schneider and R. Wattenhofer. 2010. What is the use of collision detection (in wireless networks)? In Proceedings of the 24th International Symposium on Distributed Computing (DISC'10). 133-147. DOI : https://doi.org/10.1007/ 978-3-642-15763-9_14

[45] K. M. Sivalingam, M. B. Srivastava, and P. Agrawal. 1997. Low power link and access protocols for wireless multimedia networks. In Proceedings of the 47th IEEE Conference on Vehicular Technology, Vol. 3. 1331-1335. DOI : https://doi.org/ 10.1109/VETEC.1997.605397

[46] D. E. Willard. 1986. Log-logarithmic selection resolution protocols in a multiple access channel. SIAM f. Comput. 15, 2 (1986), 468-477. DOI : https://doi.org/10.1137/0215032

Received September 2018; accepted June 2019 